\title{
Simulation of RCC Crack Growth Due to Carbon Oxidation in High-Temperature Gas Environments.
}

\author{
E. V. Titov ${ }^{*}$ D.A. Levin ${ }^{\dagger}$ \\ Pennsylvania State University, University Park, PA 16802, \\ Donald J. Picetti \\ The Boeing Company, Huntington Beach, CA 92647, \\ Brian P. Anderson \\ $N A S A(J S C-E G 311)$
}

The carbon wall oxidation technique coupled with a CFD technique was employed to study the flow in the expanding crack channel caused by the oxidation of the channel carbon walls. The recessing $3 \mathrm{D}$ surface morphing procedure was developed and tested in comparison with the arcjet experimental results. The multi-block structured adaptive meshing was used to model the computational domain changes due to the wall recession. Wall regression rates for a reinforced carbon-carbon ( $\mathrm{RCC}$ ) samples, that were tested in a high enthalpy arcjet environment, were computationally obtained and used to assess the channel expansion. The test geometry and flow conditions render the flow regime as the transitional to continuum, therefore Navier-Stokes gas dynamic approach with the temperature jump and velocity slip correction to the boundary conditions was used. The modeled mechanism for wall material loss was atomic oxygen reaction with bare carbon. The predicted channel growth was found to agree with arcjet observations. Local gas flow field results were found to affect the oxidation rate in a manner that cannot be predicted by previous mass loss correlations. The method holds promise for future modeling of materials gas-dynamic interactions for hypersonic flight.

\section{Introduction}

The reinforced carbon-carbon (RCC) nose cup and wing leading edge of the Space Shuttle, studied experimentally and numerically in this research, are vulnerable to cracks that can be formed through micrometeoroid and orbital debris impact. ${ }^{1}$ During the Earth atmosphere re-entry, the free stream species, including reactive atomic $\mathrm{O}$, may penetrate through the bare carbon-crack channel and oxidize the channel

* Post Doctoral Scholar, Department of Aerospace Engineering, AIAA member.

${ }^{\dagger}$ Professor, Department of Aerospace Engineering, AIAA Associate Fellow. 
walls. The resulting growth of the crack can pose severe structural damage to the wing and the nose portion of the vehicle. The dimensions of the cracks are sufficiently small while the flow presiding the channel has larger characteristic sizes. This is why for in-flight re-entry conditions, as well as ground based testing, the multi-scale flow solutions should be obtained.

The crack expansion process in RCC samples, modeling a portion of the Space Shuttle wing, has been studied in ground-based arcjet tests ${ }^{2}$ and the high velocity meteoroid impact holes subjected to the arcjet flow conditions were presented in. ${ }^{3}$ The purpose of this work is to demonstrate a physical model that may be employed in numerical simulations of microflows through a carbon channel and to predict the extent of the channel expansion in the geometrically complex configurations including 3D Space Shuttle leading edge fragment and axisymmetric stagnation region hole which includes a sub-channel causing delamination. Atomic oxygen impacting a bare carbon wall was assumed to chemically react with the channel walls to produce $\mathrm{CO}$ gas that then enters the flowfield. The probability of the reaction is the only free parameter in the model, with a value of 0.9 used in all simulations. ${ }^{4}$

\section{Experiment Conditions}

The high energy flow is generated in the arcjet facility through an expansion of the heated and pressurized gas through a supersonic nozzle. Figure 1 presents the stagnation point hole experimental setup, which is referred to as Case 1151 in this paper and Figure 2 presents the schematics of the experimental facility along with the major flow and device parameters as well as sub-zones for NATA and CFD (Gasp) ${ }^{5}$ solutions for the case referred to as Case 2033. The specimen presented on the right hand side of the Fig. 2 resembles a portion of the Space Shuttle leading edge with a tiny channel machined through the surface which is exposed to the flow. The initial (left hand side) and the post-test (right hand side) configurations of the specimen Case 2033 are presented in Fig. 3. It is clearly visible in the figure that the initial micro-channel has significantly expanded, when subjected to the arcjet flow conditions similar to that during the vehicle Earth atmosphere re-entry. Similar observation is evident for the case 1151. If happened during the flight, the damage of such a magnitude may severely compromise the wing structure by exposing its interior to the high energy re-entry flow. The goal of this research is therefore to develop and test a computational technique which can predict the microchannel expansion when subjected to the chemically active re-entry flows.

\section{The Computational Technique}

In order to model the channel expansion, a CFD solver should be enabled with the specific boundary conditions modeling the chemical processes at the walls of the channel. Because of its robustness, when used for the solutions of the high and low speed compressible flows, the Gasp ${ }^{5}$ N-S based solver was used in this research. An additional type of boundary conditions previously not available in GASP was implemented based on the below explained formulation. 
An important feature of the crack flow modeling is the interaction between the gas and the channel wall, which leads to mass, momentum and energy exchange. It was found that the chemical reactions among gas species (such as $\mathrm{O}+\mathrm{N}_{2} \rightarrow \mathrm{NO}+\mathrm{N}$ ) are rare in the channel flow since the total reaction energy of the colliding molecules for a gas temperature of $4000 \mathrm{~K}$ is usually less than the chemical activation energy. As is discussed in Ref., ${ }^{6}$ atomic oxygen plays an important role in carbon mass surface loss. Reference ${ }^{4}$ gives a probability close to 0.9 for the gas surface reaction,

$$
\mathrm{C}_{(\mathrm{s})}+\mathrm{O} \rightarrow \mathrm{CO}
$$

where, the temperature of gaseous atomic $\mathrm{O}$ is about $1500 \mathrm{~K}, \mathrm{C}_{(\mathrm{s})}$ is a carbon atom at the wall surface, and the other species are gaseous. We can estimate the mass loss due the above gas-surface reaction in a simple calculation. As the gas travels through the channel, it interacts with the carbon wall. The number flux normal to the channel wall, $\dot{N}$, is given as, ${ }^{7}$

$$
\dot{N}=\frac{n_{\mathrm{O}}}{2 \sqrt{\pi} \beta}
$$

where $n_{\mathrm{O}}$ is the atomic $\mathrm{O}$ number density and $\beta$ is the reciprocal of the molecular most probable thermal velocity. The mass loss, surface regression rate, $\dot{S}$, is related to $\dot{N}$ by,

$$
\dot{S}=\frac{\dot{N}}{n_{C}} P
$$

where $n_{C}$ is the carbon number density (a mass density value of $1.575 \mathrm{gm} / \mathrm{cm}^{3}$ was assumed) and $P$ is the reaction probability for the $\mathrm{C}_{(s)}+\mathrm{O}$ reaction. Using typical values in the boundary layer region of the channel flowfield of $n_{\mathrm{O}}=3.0 \times 10^{21} \mathrm{~m}^{-3}, T=2400 \mathrm{~K}$, and a reaction probability of 0.9 , we obtain an approximate surface regression rate of $0.02 \mathrm{~mm} / \mathrm{s}$. This wall regression rate will be shown to be typical of the values measured in the arcjet testing.

The typical magnitude of the wall regression rate, when compared with the channel dimensions (from 1 to $50 \mathrm{~mm}$ ), suggests that the material response and the gas dynamic flows may be loosely coupled, thereby simplifying the crack wall damage modeling. Since it takes the gas less than $10^{-3} \mathrm{sec}$ to travel through the channel, it is reasonable to assume that the flow through the channel reaches a steady state at each of the various, time-dependent channel geometries.

Modified boundary conditions which account for the processes given by Eq. (1) were developed for the GASP commercial CFD software which solves N-S equations. The numerical solution of the Navier-Stokes equations for viscous flow was obtained by use of the finite volume discretization of the computational domain done with the Gridpro $^{8}$ meshing software. The temperature jump and velocity slip corrections to the boundary conditions were used to account for the rarefaction effects inside the channel. 


\section{Computational Domain and expanding surface morphing}

\section{A. Topology based meshing}

In general, block structured meshing is a well defined notion - meaning that the entire computational domain is divided into blocks and each of the blocks has a structured mesh. Since topology is a more abstract notion let us define it.

In the geometrical sense, topology is defined as an organization of space. For example one can think of geometrical figures as topology elements, but not all geometrical figures represent distinct topology types. If we take for example a torus with some size as a topology example and stretch or bend it, or do whatever else we want to do with its shape and characteristic sizes, the resulting geometrical figure will not represent a new topology as long as our movements do not make the torus surfaces intersect each other. In this sense, a torus and say a picture frame are topologically equivalent. The concept behind Gridpro ${ }^{8}$ meshing rests on the idea that a topology remains unchanged even though the shape of the corresponding geometrical entities (blocks of mesh in this case) change to some extent as explained above. Topology usage for meshing has some specifics. Let us cover some of them.

In order to mesh the volume regions, one must cover them with wireframe topologies that approximately follow their surface boundaries. The majority of Gridpro ${ }^{8}$ operations deal with creating and modifying topologies. We note that such topologies consist of a wireframe and surface assignments. The wireframe consists of topology nodes and edges. The assignment process is a way for telling the Gridpro ${ }^{8}$ software to which particular boundary one or another piece of topology belongs to.

The next issue is how accurately the user should position the topology elements (nodes and edges). Gridpro $^{8}$ can work with loosely positioned topologies. In other words, it can grid a topology which is defined with nodes and edge positions that only roughly conform to the desired (corresponding) geometry. A wireframe topology does not have to exactly fit the boundaries for the region to be meshed. However, the topology itself (the way the topology elements are assembled and assigned to the surfaces) must not have errors and if an error in the topology is made the meshing will not proceed. If a topology element (a node or an edge) is positioned too far from a surface, this will not create a fatal error but the meshing process will take longer than it otherwise should. Thus, as long as the topology is positioned with some care, the surfaces and volumes will be meshed with relative ease. There is no a priory way to tell how close the topology elements should be placed with respect to the corresponding surfaces. The trade off is that the closer a topology resembles the actual geometry, the faster the meshing procedure will converge. The final mesh quality does not depend on how close the topology elements are located to the corresponding surfaces, but again, errors in the topology, or a topology which does not properly reflect the underlying computational domain, may result in a fatal problem during the meshing procedure or a mesh of poor quality.

Gridpro $^{8}$ grids flow volumes in both two and three dimensions and therefore topologies can be two and three dimensional in Gridpro. The 1151 case is solved in two dimensions while 2033 is a full 3D case. However some initial modeling of the 3D problems as 2D may help building a better topology and the resulting mesh. 
In two dimensions, gridded blocks are represented by convex quadrilaterals, or quads and in three dimensions, gridded blocks are represented by convex hexahedrons, or hexes.

After this short introduction to the topology as it is used in Gridpro, let us consider the 1151 case and decompose its computational domain into topology quads and then mesh the domain. Because surfaces can be complex, they may require complex topologies and unfortunately no unique topology exist for a case. Furthermore different topologies will produce meshes of different quality. Let us now start with the simplest topology possible for the case 1151 and see the results. Figure 4 presents such a topology consisting of 18 topology elements and 14 topology nodes. As can be seen the topology presented in Fig. 4 roughly mimics the domain boundaries and physical surfaces presented in Fig. 5, however two observations should be done. The first one is that the computational domain represented by the 2D surfaces in Fig. 5 exactly describes the specimen and the outer domain boundaries while the topology presented in Fig. 4 has a similar shape but its edges and nodes do not necessarily lay on the surfaces. The second observation is that even this simple topology contains some additional edges and nodes which are not required to define the domain boundaries and body surfaces. These edges and nodes are created keeping in mind the extended structure of the mesh blocks and are in fact required. The topology nodes are assigned to the corresponding surfaces, which in this case is rather obvious and one proceeds with the meshing. Recall that assignment of a node to an edge is used to connect the wireframe topology to the true physical surfaces of the computational domain.

Figure 6 presents the resulting mesh block structure obtained after a few hundred steps of the meshing procedure solving the variational equations as defined by the underlying algorithm. ${ }^{8}$ As can be seen in the figure the resulting block structure is topologically equivalent to the wireframe structure presented in Fig. 4, however the outer boundaries of the mesh blocks now exactly matching the boundaries of the 1151 specimen and the computational domain. The inner boundaries of the mesh blocks have also been modified to create an optimum mesh structure based on the provided topology.

Let us take a look at the mesh itself. The mesh density is kept relatively coarse for this illustrative example to reduce clutter. Figure 7 presents the entire mesh for the case 1151 with an enlargement of the channel area presented in Fig. 8 and the corner of the channel entrance shown in Fig. 9. As can be seen in Fig. 7, the overall mesh quality is reasonably good with acceptable meshing inside the channel. Let us now look into some details. It can be seen in Fig. 9 that the mesh in this area is actually of a very poor quality. None of the quality criteria such as orthogonality or skewness can be satisfied with such a mesh. In fact if one attempts to use such a mesh for a CFD solution, the solver would probably fail or produce some unphysical results at best.

Let us see what can be done to improve the mesh quality. We start with analyzing what causes the problem which arises from the so called "singularity" at the corner of the channel. There are several types of singularities defined in Gridpro (which should not be confused with the mathematical definition of a singularity). In this case, the particular type of singularity arises because four topology edges and only two surface edges are converging at the corners of the channel.

Let us try avoiding the problem. To avoid it we define a new 1151 topology shown in Figure 10 with 
an enlargement of the channel area presented in Fig. 11. Figure 12 presents the new surfaces defining the computational domain. There are several differences between the previous and the new topology and corresponding surfaces involved in the domain modeling. The first difference is the addition of the so called artificial surfaces which can be observed in Fig. 12. These surfaces do not represent real boundaries of the computational domain. Their sole purpose is to resolve the singularity issue at the corners of the specimen. Their presence however must be reflected in the topology structure for the effect to take place. As can be seen in Figs. 10 and 11, several new topology blocks have been added corresponding to the new domain model. The topology nodes must now be partly reassigned to the new surfaces which came into picture. Some of the nodes must now have double or tripple assignments. For example the topology node located at the corner of the channel entrance (see Fig. 11) is now assigned to three surfaces: to the channel wall, the specimen outer surface, and to the artificial surface located at the entrance of the channel.

Let us now look at the resulting mesh. The block structure of the new 1151 mesh is presented in Fig. 13. It is immediately clear from the observation of the block structure that the meshing problem at the corner of the channel entrance is now resolved. The angles at the corners of the blocks are close to 90 degrees, which is a good indication of the mesh orthogonality in the problematic areas. The boundaries of the blocks are also adapted to produce a better mesh based on the new version of the topology. Let us take a look at the mesh itself. Figure 14 presents the entire mesh for the the domain while Fig. 15 presents the mesh inside the channel with an enlargement of the channel corner presented in Fig. 16. As can be seen in the figures, and, in particular in Fig. 16, the mesh quality is improved dramatically and this new mesh can definitely be used for the CFD computations. One additional implication of using the artificial surfaces is the improved ability to manipulate the local mesh density. In the 1151 case we want the mesh to be dense inside the channel to resolve the area of interest and less so in the outer portion of the domain to save computational cost. With the artificial surfaces in place such a mesh can be produced. The artificial surfaces bound the interior of the channel preventing the mesh optimizer from moving the mesh points located inside the channel into the areas in front and behind the channel.

With the acceptable mesh having been created we conclude the introduction of the topology concepts. Our resulting mesh may still need additional refinement, however. Issues such as the shock wave resolution in front of the specimen and optimization of the mesh density need to be solved before the actual CFD calculations may be done. All of these issues are addressed and will be commented in result section of this article. Cases 1151 with delamination and 2033 solved here are significantly more involved, but the presented concepts hold. Let us now introduce the additional subjects of 3D surface definitions and surface morphing, relevant to the modeling of the 2033 and 1151 cases.

\section{B. The surface expansion modeling}

In order to model the regressing walls of the 1151 and 2033 channels a surface morphing procedure which accounts for the local wall oxidation should be developed. The corresponding procedure described here rests on the assumption that initial surface is smooth (no sharp corners) and is defined as a set of adjacent quads. 
Furthermore, we do not expect the surface mesh to uniquely define the normal direction with respect to a particular surface side. The procedure is capable of creating surface meshes of a sufficiently good quality for meshing the computational domains and is as follows.

We start with a control net based channel surface created in Gridpro. We mesh the computational domain where the channel surface is one of the boundaries and run the Gasp ${ }^{5}$ N-S solver to obtain a CFD solution. A stand alone morphing code reads the initial channel surface mesh data and the CFD solution results then computes the normal surface vectors as the cross products of the surface element edges. The normals are associated and stored with each of the surface element. If nodes are shared among several surface elements (which is usually the case, as shown in Fig. 17), an averaging is done to compute the normals associated with the surface nodes. Each of the following cross-products $\mathbf{O A} \times \mathbf{O C}, \mathbf{O E} \times \mathbf{O D}$ and $\mathbf{O C} \times \mathbf{O D}$ defines a different normal direction at point $O$.

The resulting normal direction $\mathbf{O F}$ is the result of averaging. We then provide reference points whose coordinates are given with respect to the origin of the computational domain. These reference points indicate in an absolute sense the inside and outside of the channel. Based on these reference points, the directions of the normal vectors associated with each of the surface nodes are adjusted to be uniformly directed with respect to the surface side. The problem, that the provided reference points solve, is illustrated in Fig. 18. In order to define a surface mesh element it is not sufficient to specify the nodes of the surface with their coordinates. In addition to this, the connections between the nodes must also be specified. For example let the nodes of a simple surface which consists of only two quads, as shown in Fig. 18, to be counted and have the numbers from 1 to 6 as presented in the figure. Then the two quads presented in the figure can, for example, be defined as follows: quad1 ([1,4];[1,2];[2,3];[3,4]) quad2 $([4,3] ;[4,6] ;[3,5] ;[6,5])$. The pairs of numbers in the square brackets represent the edges of the quads. Let us now define a universal rule for the definition of the normal direction associated with a quad as the cross product of vectors defined by its two first edges. This would imply that the normal of the first quad is defined as follows $[1,4] \mathrm{x}[1,2]$ and for the second quad it will be $[4,3] \mathrm{x}[4,6]$. Note that we assume the edges to be vectors with directions from the first nodes of the edges to the second ones. The presented cross-products define the normals to the quads as is shown in Fig. 18. Both of the normals are defined correctly but they point to opposite directions. This would be a problem in our application of morphing of the surfaces, thus, a special care must be taken to define the normals properly. The next step is to find a closest volume node for each surface node with the values of the computational results to be associated with the surface nodes. The local oxidation rate at each surface node is computed then and stored. With the local oxidation rate and the normal direction obtained, each of the surface elements is accordingly moved. The movement of the nodes is done in a time loop incremented with the time steps that are much smaller than the time between sequential CFD runs. Visual surface mesh quality control achieved by taking the morphed mesh back into the Gridpro and checking that the resulting surface is smooth enough.

If there are imperfections due to the collapsing of the surface elements, the procedure is re-started and run up to the time where the surface mesh was still smooth. This intermediate surface mesh serves as a 
reference for an analytical approximation. After several of experimentations, it was found that a parabolic approximation to the channel surface is useful. It is defined as follows. The oxidation rates along the edges of the channel (the entrance, the exit and the corners which are defined by the intersection of the channel surface with the channel symmetry plane) are computed and stored for each of the rows and columns of the channel surface mesh. These values serve to define a series of parabolas associated with each of the rows and columns of the surface mesh. Figure 19 shows two examples of parabolic curves for a row and a column at the middle of the surface mesh. For this specific case, there are therefore 57 times 238 parabolic surface curves which are re-computed every time the surface is morphed, or every $1 \mathrm{sec}$.

The surface regression is then performed in two steps. We first morph each surface mesh point by moving it half of the local computed CFD defined oxidation rate from the parabolic expression for the row that it belongs to. We then repeat the procedure again for each surface mesh point but now move it from the parabolic value depending on its associated column. In such a way we take into account the rate along all of the channel corners when computing the amount of necessary displacement for each of the surface mesh points. This gives a more realistic and a more 3-D meshing friendly approximation of the surface than a surface advancement based on the actual local CFD oxidation rates. However this is not the final mesh which can be directly used in the 3-D volume meshing, since it is not integrated with the rest of the surfaces which define the boundaries of the specimen. The resulting mesh is taken back into Gridpro and is used as a control net to produce the spline surface to be integrated with the outer surface of the specimen. Finally the spline based surface mesh is used in Gridpro to account for the channel surface mesh change, and a new 3 -D volume computational mesh is created.

\section{Results and Discussion}

\section{A. Case 1151 with delamination meshing and flow results}

Attention was paid to the proper meshing of the computational domain making use of the Gridpro software. The multi zone layout of the domain presented in Fig. 20 allowed for the adjustment of the zone boundaries to automatically improve the mesh quality in terms of its orthogonality skewness and aspect ratio. To account for the possible influence of delamination, a sub-channel was added to the layout of the computational domain as shown in Fig. 21. After a preliminary CFD solution, based on a coarser mesh, the position of the shock wave was determined and the mesh was adapted to better capture the shock wave gradients as presented in Fig. 22. To better resolve the boundary layer gradients mesh points were clustered at the walls as presented in Fig. 23. It can be seen that the mesh cells are orthogonal with small aspect ratio (excluding the cells in the boundary layer) and with almost no skewness in 80 to $90 \%$ of the cells.

To resolve mesh singularities at the corners of the specimen and at the entrance of the sub-channel, artificial double-sided surfaces were introduced into the computational model at the mesh generation stage. After each of the sequential computations, the channel walls and adjacent boundaries (including artificial walls) were morphed and the new computational domain was re-meshed in a semi-automated manner. Mesh 
quality was checked for skewness with tools available in Gridpro for each of the solved cases (see Fig. 24). The mesh gradients are extremely large in the cases with a sub-channel since the outer flow, channel flow, and the sub-channel flow were all solved in a single run starting from the pre-shock NATA solution. However, the mesh quality was never allowed to worsen below the strict limits imposed by the checking procedures. After morphing the surface, the re-meshing of the computational domain was relatively straightforward with some limited amount of manual work.

\section{B. Influence of delamination}

In order to understand the behavior of the flow parameters influencing the wall regression rate, temperature and atomic oxygen mass fraction are presented in Figs. 25 and 26. Additionally CO mass fraction and velocity streamlines are presented in Figs. 27 and 28. Figure 29 presents a comparison between the oxidation rates at the channel wall for 0 and 50 seconds. Finally Fig. 30 presents a comparison between the computational results for calculations with and without the sub-channel with the experimental data. Figures $25-28$ show that for the expanded sub-channel the flow in the sub-channel entrance area stagnates with recirculation areas observed. This results in the atomic oxygen available in the sub-channel area being consumed by the chemically active wall. $\mathrm{CO}$, the product of the oxidation, remains in the sub-channel entrance area shielding a fresh intake of the atomic oxygen from the channel flow. This in turn results in a lower oxidation rate in the sub-channel area compared with that along the main channel wall as can be seen in Fig. 29. Figure 29 demonstrates that this occurs because the $50 \mathrm{sec}$ surface regression profile is actually reversed compared to the initial $(0 \mathrm{sec}$.) result. The phenomena is probably due to two different factors contributing to the oxidation rate: pressure build up at the sub-channel corners and the stagnation of the flow in the sub-channel area. These factors influence the oxidation rate magnitude in different directions. A more accurate DSMC or BGK solution to the sub-channel inlet problem may provide more insight into the phenomena.

The entrance and exit hole diameters growth, presented in Fig 30, show the influence of the delamination (the presence of the sub-channel) on the main channel regression. As can be seen in the figure, the presence of the sub-channel increases the rate of the exit diameter growth compared with the results of the computation without the sub-channel. This influence brings the computational results closer to the experimental data. At the same time, the growth of the entrance diameter of the channel remains the same (does not depend on the presence of the sub-channel).

\section{Influence of the flow chemistry upstream of the channel}

Prior to presenting the influence of the flow chemistry upstream of the channel on the wall regression rates some explanation must be made about the accuracy of the attempted modeling. Figures 31 and 32 present the temperature profiles in the shock wave and along the stagnation line respectively. The locations in the flow where the temperature profiles presented in Figs. 31 and 32 are taken from are shown in Figs. 33

and 34 respectively. As can be seen in the figures the temperature levels at around 9,000 K, a temperature sufficiently high that chemical non-equilibrium must be considered in the gas dynamics. 
Our consideration of the flow chemistry is based on a finite rate model at a single temperature which is not very accurate at these temperatures. However we will show that the atomic oxygen generation in the shock wave can influence the wall regression rate to some extent. For this reason a comparison of the wall regression rates between the N-S solutions with and without chemistry in the flow are presented below.

Comparisons between the temperatures and mass fractions of atomic oxygen (the two parameters which are included into the computation of the wall regression rate) are presented in Figs. 35 and 36 as top and bottom figures for cases with and without flow chemistry. Additional comparison of pressures and temperatures are presented in Figs. 37 - 38. The set of chemical reactions used in this research is given in Table 2 with reaction coefficients being dependent on the temperature as defined in the GASP $^{5}$ computational tool. The results are provided for an initial channel configuration geometry. It can be seen in the figures that the general flow parameters (namely pressure, temperature and $\mathrm{x}$ component velocity) have reasonable spatial distributions. In particular, flow pressure decreases while the flow accelerates in the channel and the temperature of the flow drops because the thermal energy is transformed into the kinetic energy of the accelerating flow. It should be noted that some difference exists between the cases with and without flow chemistry. The temperature after the shock is smaller in the case with chemical reactions due to dissociation reactions which consume thermal energy. The main point with respect to this work is that amount of atomic oxygen in the flow is slightly higher in the case with chemical reactions due to the additional production of atomic oxygen by dissociation of the oxygen molecules in the shock. Examination of Fig. 26 shows that the atomic oxygen mass fraction does not change inside the channel, since there are essentially no chemical reactions there, as was discussed earlier in the paper.

The above phenomena is reflected in Figs. 39 and 40. The somewhat increased amount of the atomic oxygen increases the wall oxidation rate which is reflected in the amount of carbon monoxide production as presented in Figs. 39 and 40 for the entire computational domain and in the channel area, respectively. A comparison between the computational results with and without chemistry and the experiment is presented in Fig. 41. For reference, the results for the flow without the sub-channel are also presented. As can be seen in the figure, the production of the atomic oxygen in the flow influences the wall regression rate at the entrance of the channel, where the flow is exposed to the shock generated atomic oxygen. Unlike the earlier result (Fig. 30) which showed that the presence of the sub-channel primarily affects the outlet diameter, here the additional atomic oxygen strongly affects the channel entrance region oxidation rate.

\section{Case 2033 meshing and flow results}

Figure. 42 provides a schematics of the three-dimensional wedge case geometry and the layout of the computational domain associated with it. Presented in the figure is the 320 second case, with the channel already substationally expanded to demonstrate all of the features of the computational domain. The initial channel dimensions were however $0.990 \times 38.1 \mathrm{~mm}(0.0387 \times 1.5$ inches $)$ with a depth of $6 \mathrm{~mm}$. The multi-scale external-internal flow for this setup was solved in a single run. The computational domain was structurally meshed using the block-structured techniques available in Gridpro with different mesh densities in different 
blocks in order to accommodate the flow phenomena which ranged from the shock wave structure in front of the modeled portion of the Space Shuttle wing leading edge to the boundary layer inside the micro-channel crack. The computational domain consisted of the larger outer sub-domain and a tiny well meshed microchannel domain. Figure. 42 also presents the mesh block structure which was optimized to produce a high quality 3D mesh covering the entire computational domain. The inlet surface presented in the figure was set up with the boundary conditions corresponding to the free stream NATA pre-shock solution presented in table 3. The no-slip thermal boundary conditions were set at the surface of the specimen, which is marked as wedge in the figure, while the temperature jump velocity slip boundary conditions which accounted for the oxidation at the wall were used at the channel walls. First order extrapolation boundary conditions were set at the two exits of the computational domain, and two symmetry planes were used to limit the computational domain in $\mathrm{Z}$ direction. The flow direction is also presented in the figure as a vector in the XY plane, making 45 degrees angle with the $\mathrm{X}$ axis. Figure 43 presents the computational mesh. As can be see in the figure, care was taken to resolve the flow features especially those inside the channel and in vicinity of its entrance. Figure 44 further illustrates the features of the computational domain by presenting the Mach number contours obtained from a CFD solution with use of the presented computational mesh.

To better illustrate the channel portion of the computational domain, a mesh block which corresponds to the channel and its entrance area is presented in Fig. 45. As can be seen in the figure, several mesh blocks were used to mesh the channel area, which required, in part, the boundary layer features resolution, since the flow properties at the walls of the channel are essential for the correct prediction of the channel expansion. Figure 46 presents the atomic oxygen density contours plotted in three dimensions using the excerpt of the computational mesh, presented in Fig. 45.

To illustrate the 3D shock wave capturing attempted in this research, a portion of the computational domain with the exposed interior of the mesh is presented in Fig. 47. This is a 60 second case mesh which was used in one of the solutions from the series of solutions covering the expansion of the channel. As can be seen in the figure, the mesh resolution is significantly higher in the shock wave area which allows for a better shock wave structure modeling. The quality of the solution generated based on this mesh is presented in Fig. 48 where the temperature contours are presented in 3D space, showing, in particular, the shock wave capturing. The series of N-S calculations was performed based on the computational domains similar to the one presented in Fig. 42. The free stream uniform boundary conditions obtained from the NATA solution (see Table 3) were applied along the entrance of the computational domain as presented in Fig 42. The computational domain accommodates bow and oblique shock waves in front of the specimen. The inlet surface is aligned with the shock wave structure to reduce the size of the computational domain.

A series of iterations at $0,60,120,220,320$ and 420 seconds were performed with the channel shape changing according to the oxidation process at the wall.

Figures 49- 51 show the major flow features in the computational domain, namely Mach number, pressure and temperature contours. As can be seen in the figures the overall flow features are well resolved and show a reasonable pattern in all portions of the computational domain. In particular, the shock wave structure is 
resolved and the flow inside the channel is accurately modeled. Figures 52 and 53 show the atomic oxygen and CO mass fraction distribution at the channel walls and down stream (over the specimen) for the 60 sec case. As can be seen in the figures, a small portion of the product of reaction at the walls of the channel escapes the channel through the subsonic boundary layer and moves over the specimen. A corresponding decrease in the atomic oxygen mass fraction can also be observed in Fig. 52. Such a mechanism can actually reduce the specimen surface destruction in the downstream area since the product of reaction, the $\mathrm{CO}$ generated inside the channel, when escaping the channel, may shield the the specimen surfaces from the chemically active atomic oxygen.

Figure 54 presents the shape of the channel computed at different times. During the first two computational steps, 0 and 60 seconds, the surface morphing procedure was used in full as described in Sec. B. After the channel was sufficiently opened with its walls exposed to the coming flow, more reliable values of the local oxidation rates become possible to compute. Beginning with 120 case, the parabolic surface approximation as defined in section B was omitted and the local surface displacements were computed based on the local oxidation rates obtained from the CFD solutions. The rest of the procedure described in section $\mathrm{B}$ remained the same.

Following observations can be made from the results of modeling. The initial channel expansion is less intensive and more even in all of the directions. However, when the channel opens, which allows more atomic oxygen to enter the channel interior, the process accelerates, with the predominant expansion areas being formed in the downstream portion of the channel.

Figure 55 shows a comparison between the channel shapes obtained computationally and experimentally in the arcjet facility. The experimentally obtained surface was roughly reconstructed based on the post test measurements of the channel. As can be seen in the figure the channel sizes are in a good agreement at the entrance of the channel with the experimentally obtained channel being slightly larger. The size of the experimental channel at the exit is approximately 20 percent smaller than that of the computational one. The overall shapes of the channels are remarkably close.

\section{Conclusions}

In this work, an approach was developed to model the continuum flow through the carbon crack channel of the RCC test samples. The gas dynamics was modeled using the N-S technique with chemically active boundary conditions. Atomic oxygen impacting a bare carbon wall is assumed to chemically react with the wall to produce $\mathrm{CO}$ gas (with a probability of 0.9 ) that enters the flowfield. It was found that carbon surface degradation caused by atomic oxygen accounts for large mass loss and shape similar to the arcjet samples for considered cases. 


\section{Acknowledgments}

E. V. Titov and D. A. Levin would like to acknowledge support from Boeing contract No. $6 \mathrm{H} 08845$ and NASA Grant NNX08AD84G from NASA/Johnson Space Flight Center. We would especially like to thank the AeroSoft Corporation, Blacksburg, VA for their technical support and assistance in incorporating the gas wall oxidation boundary condition.

\section{References}

${ }^{1}$ Curry, D. M., Pham, V. T., Norman, I., and Chao, D. C., "Oxidation of Hypervelocity Impacted Reinforced CarbonCarbon," Journal of Spacecraft and Rockets, Vol. 37, No. 3, 2000, pp. 310-317.

${ }^{2}$ Lewis, R., "Quick look report," Atmospheric Reentry Materials and structures (ARMSEF), January 2004.

${ }^{3}$ Curry, D. M., Pham, V. T., Norman, I., and Chao, D. C., "Oxidation of Reinforced Carbon-Carbon Subjected to Hypervelocity Impact, NASA/TP-2000-209760," March 2000.

${ }^{4}$ Rosner, D. and Allendorf, H. D., "High-Temperature kinetics of Graphite Oxidation by Dissociated Oxygen," AIAA Journal, Vol. 3, August 1965, pp. 1522-1523.

${ }^{5}$ Gasp, The General Aerodynamic Simulation Program, Computational Flow Analysis Software for the Scientist and Engineer, User's Manual, Aerosoft Co., Blacksburg, 1996.

${ }^{6}$ Curry, D., Johansen, K., and Stephens, E., "Reinforced Carbon-Carbon Oxidation Behavior in Convective and Radiative Environments," NASA Technical paper 1284, 1978.

${ }^{7}$ Bird, G. A., Molecular Gas Dynamics and the Direct Simulation of Gas Flows, Clarendon Press, Oxford, 1994.

${ }^{8}$ Eiseman, P. R., "Adaptive grid generation," Computer methods in applied mechanics and engineering, March 1987, pp. 321-376.

Table 1. Free stream flow parameters from NATA arcjet solution and initial channel sizes for case 1151.

\begin{tabular}{ll}
\hline Channel diameter $(\mathrm{m})$ & 0.00325 \\
Channel length $(\mathrm{mm})$ & 0.006 \\
Pressure (Pa) & 70.5 \\
Mach & 5.83 \\
Temperature (K) & 900 \\
Chemical species mass fractions & $\mathrm{N}_{2}=0.298$ \\
& $\mathrm{O}_{2}=0.2971 \mathrm{e}-6$ \\
& $\mathrm{NO}=0.3763 \mathrm{e}-6$ \\
& $\mathrm{~N}=0.4666$ \\
& $\mathrm{O}=0.2345$ \\
Channel wall BC & Diffuse, oxidation reaction \\
\hline \hline
\end{tabular}


Table 2. Chemistry.

\begin{tabular}{|c|c|c|c|c|c|c|}
\hline \multirow[t]{2}{*}{ Reaction } & \multicolumn{3}{|l|}{ Forward rates * } & \multicolumn{3}{|c|}{ Equilibrium rates $* *$} \\
\hline & $C$ & $\nu$ & $-\varepsilon / k T$ & $C$ & $\nu$ & $-\varepsilon / k T$ \\
\hline $\mathrm{N}_{2}+\mathrm{N}_{2} \rightarrow \mathrm{N}+\mathrm{N}+\mathrm{N}_{2}$ & $4.7 \times 10^{14}$ & -0.5 & 113000 & 18000 & 0 & 113000 \\
\hline $\mathrm{N}_{2}+\mathrm{O}_{2} \rightarrow \mathrm{N}+\mathrm{N}+\mathrm{O}_{2}$ & $1.9 \times 10^{14}$ & -0.5 & 113000 & 18000 & 0 & 113000 \\
\hline $\mathrm{N}_{2}+\mathrm{NO} \rightarrow \mathrm{N}+\mathrm{N}+\mathrm{NO}$ & $1.9 \times 10^{14}$ & -0.5 & 113000 & 18000 & 0 & 113000 \\
\hline $\mathrm{N}_{2}+\mathrm{N} \rightarrow \mathrm{N}+\mathrm{N}+\mathrm{N}$ & $4.085 \times 10^{19}$ & -1.5 & 113000 & 18000 & 0 & 113000 \\
\hline $\mathrm{N}_{2}+\mathrm{O} \rightarrow \mathrm{N}+\mathrm{N}+\mathrm{O}$ & $1.9 \times 10^{14}$ & -0.5 & 113000 & 18000 & 0 & 113000 \\
\hline $\mathrm{O}_{2}+\mathrm{N}_{2} \rightarrow \mathrm{O}+\mathrm{O}+\mathrm{N}_{2}$ & $7.2 \times 10^{15}$ & -1 & 59500 & $1,200,000$ & -0.5 & 59500 \\
\hline $\mathrm{O}_{2}+\mathrm{O}_{2} \rightarrow \mathrm{O}+\mathrm{O}+\mathrm{O}_{2}$ & $3.24 \times 10^{15}$ & -1 & 59500 & $1,200,000$ & -0.5 & 59500 \\
\hline $\mathrm{O}_{2}+\mathrm{NO} \rightarrow \mathrm{O}+\mathrm{O}+\mathrm{NO}$ & $3.6 \times 10^{15}$ & -1 & 59500 & $1,200,000$ & -0.5 & 59500 \\
\hline $\mathrm{O}_{2}+\mathrm{N} \rightarrow \mathrm{O}+\mathrm{O}+\mathrm{N}$ & $3.6 \times 10^{15}$ & -1 & 59500 & $1,200,000$ & -0.5 & 59500 \\
\hline $\mathrm{O}_{2}+\mathrm{O} \rightarrow \mathrm{O}+\mathrm{O}+\mathrm{O}$ & $9.0 \times 10^{16}$ & -1 & 59500 & $1,200,000$ & -0.5 & 59500 \\
\hline $\mathrm{NO}+\mathrm{N}_{2} \rightarrow \mathrm{N}+\mathrm{O}+\mathrm{N}_{2}$ & $3.9 \times 10^{17}$ & -1.5 & 75500 & 4000 & 0 & 75500 \\
\hline $\mathrm{NO}+\mathrm{O}_{2} \rightarrow \mathrm{N}+\mathrm{O}+\mathrm{O}_{2}$ & $3.9 \times 10^{17}$ & -1.5 & 75500 & 4000 & 0 & 75500 \\
\hline $\mathrm{NO}+\mathrm{NO} \rightarrow \mathrm{N}+\mathrm{O}+\mathrm{NO}$ & $7.8 \times 10^{17}$ & -1.5 & 75500 & 4000 & 0 & 75500 \\
\hline $\mathrm{NO}+\mathrm{N} \rightarrow \mathrm{N}+\mathrm{O}+\mathrm{N}$ & $7.8 \times 10^{17}$ & -1.5 & 75500 & 4000 & 0 & 75500 \\
\hline $\mathrm{NO}+\mathrm{O} \rightarrow \mathrm{N}+\mathrm{O}+\mathrm{O}$ & $7.8 \times 10^{17}$ & -1.5 & 75500 & 4000 & 0 & 75500 \\
\hline $\mathrm{O}+\mathrm{N}_{2} \rightarrow \mathrm{N}+\mathrm{NO}$ & $7.0 \times 10^{1} 0$ & 0 & 38000 & 4.5 & 0 & 37500 \\
\hline $\mathrm{NO}+\mathrm{O} \rightarrow \mathrm{N}+\mathrm{O}_{2}$ & $3,200,000$ & 1 & 19700 & 0.00333 & 0.5 & 16000 \\
\hline
\end{tabular}

* Presented are the coefficients in the formula: $K_{f}=C T_{a}^{\eta} e^{-\varepsilon / k T}$

where: $k$ is Boltzmann constant, $T_{a}$ is the rate controlling temperature defined as follows:

$T_{a}=T^{\alpha} T_{\nu}^{\beta} T_{e}^{1-\alpha-\beta}$ where: $T_{\nu}$ - is vibrational temperature and $T_{e}$ is electron temperature. In the solved cases: $\alpha=1, \beta=0$

** Presented are the coefficients in the formula: $K_{c}=C T^{\eta} e^{-\varepsilon / k T}$

$\varepsilon / k T$ is activation energy 
Table 3. Flow parameters for the case 2033 (obtained from NATA arcjet solution).

\begin{tabular}{ll}
\hline $\mathrm{N}_{2}$ & 0.177 \\
$\mathrm{O}_{2}$ & 0.033 \\
$\mathrm{~N}$ & 0.551 \\
$\mathrm{O}$ & 0.239 \\
$\mathrm{NO}$ & $2.426 \mathrm{e}-6$ \\
Temperature $(\mathrm{K})$ & 929 \\
Pressure $(\mathrm{Pa})$ & 151.07 \\
\hline
\end{tabular}

** Free stream conditions taken from a separate

arcjet CFD calculation upstream of the arcjet shock.

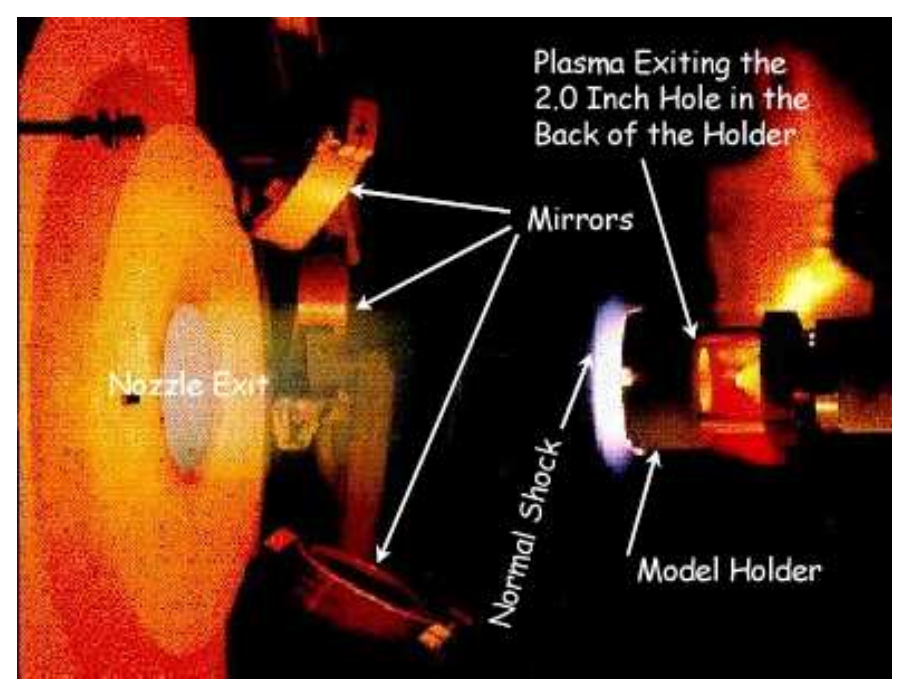

Figure 1. Case 1151. The experimental facility.

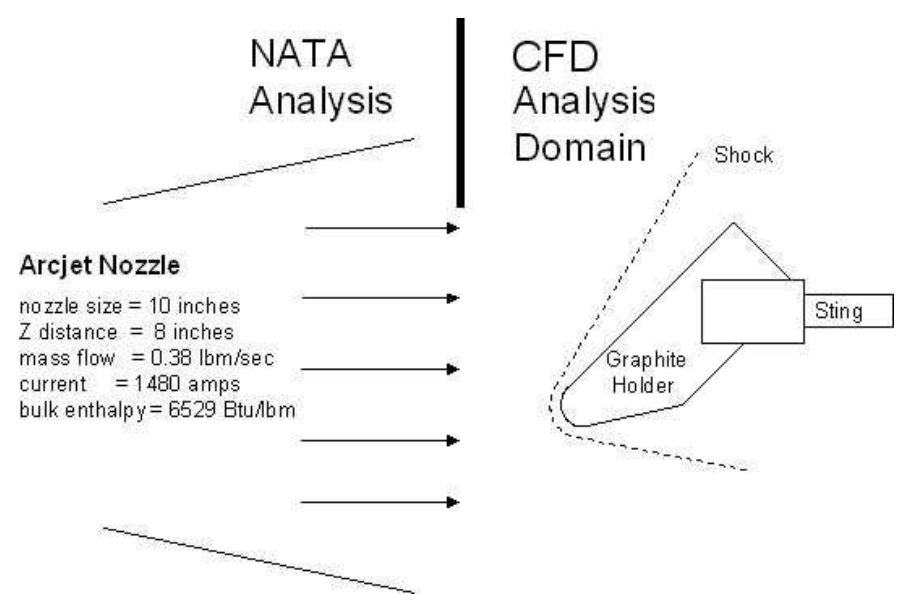

Figure 2. Case 2033. The schematics of the experimental facility. 

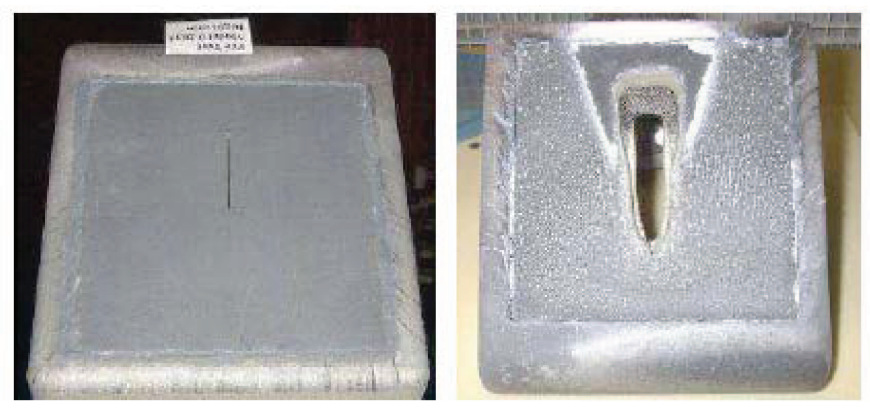

Figure 3. Initial and post-test specimen configurations.

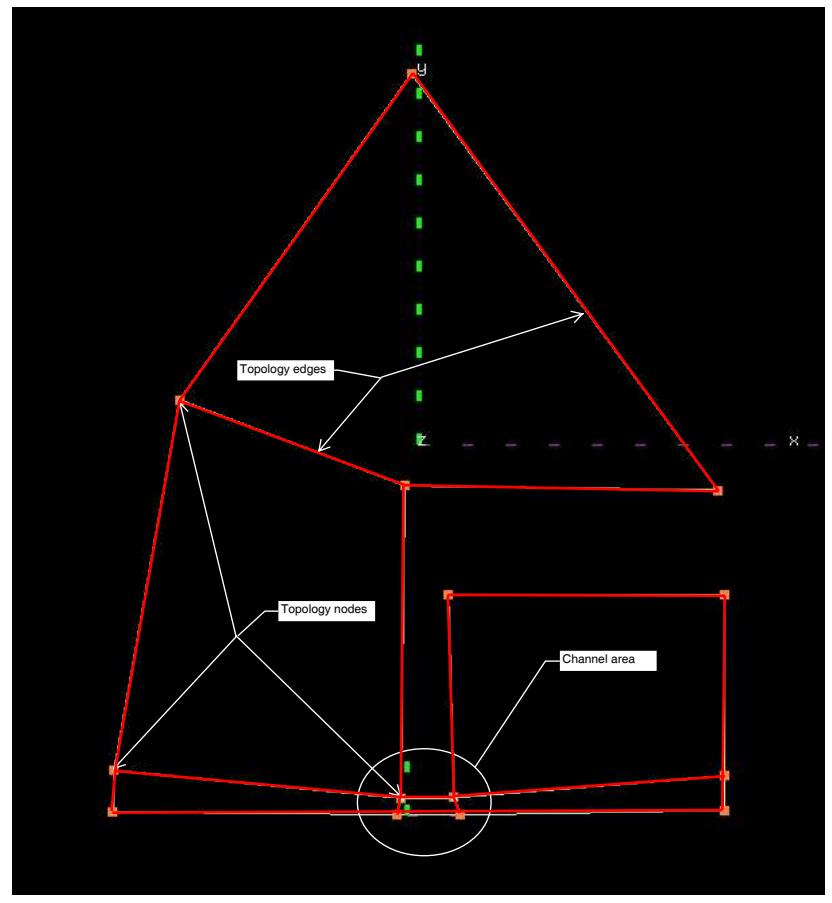

Figure 4. The simplest 1151 case topology. 


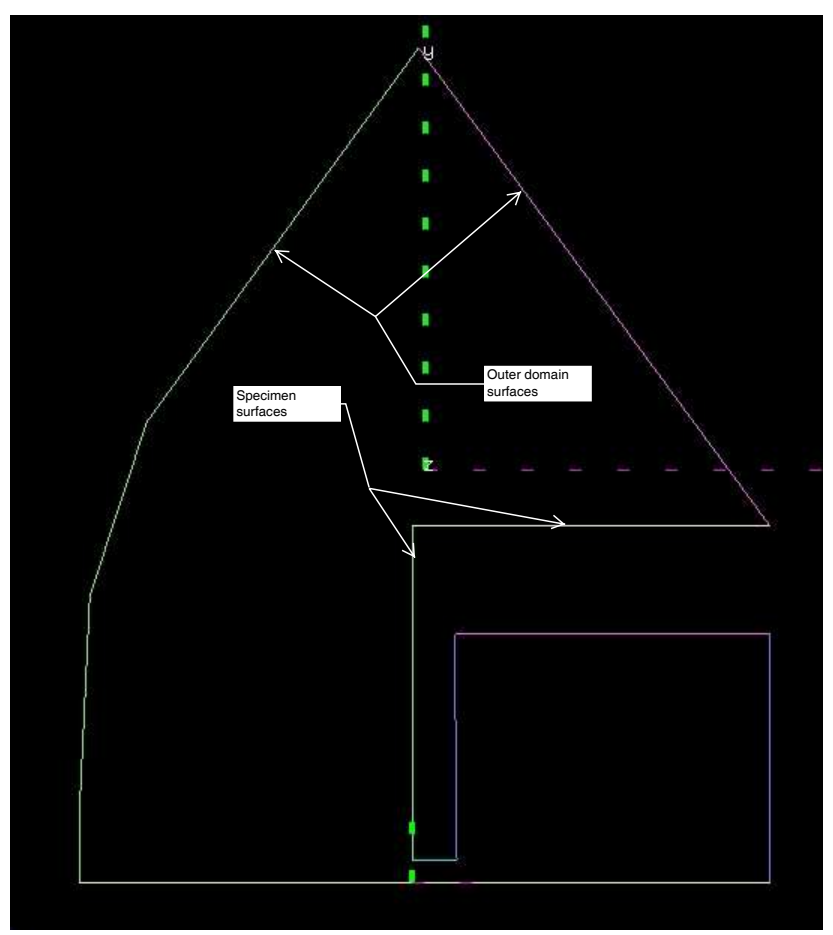

Figure 5. The simplest 1151 specimen and domain boundaries.

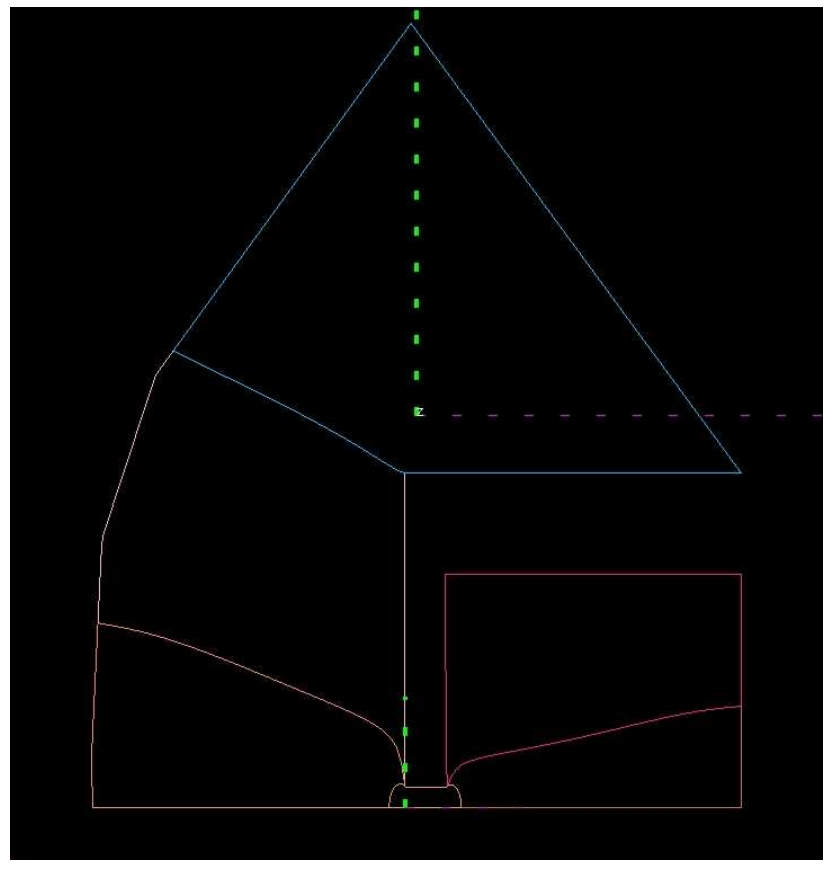

Figure 6. The simplest 1151 mesh block structure. Lines indicate the morphed topology edges. 


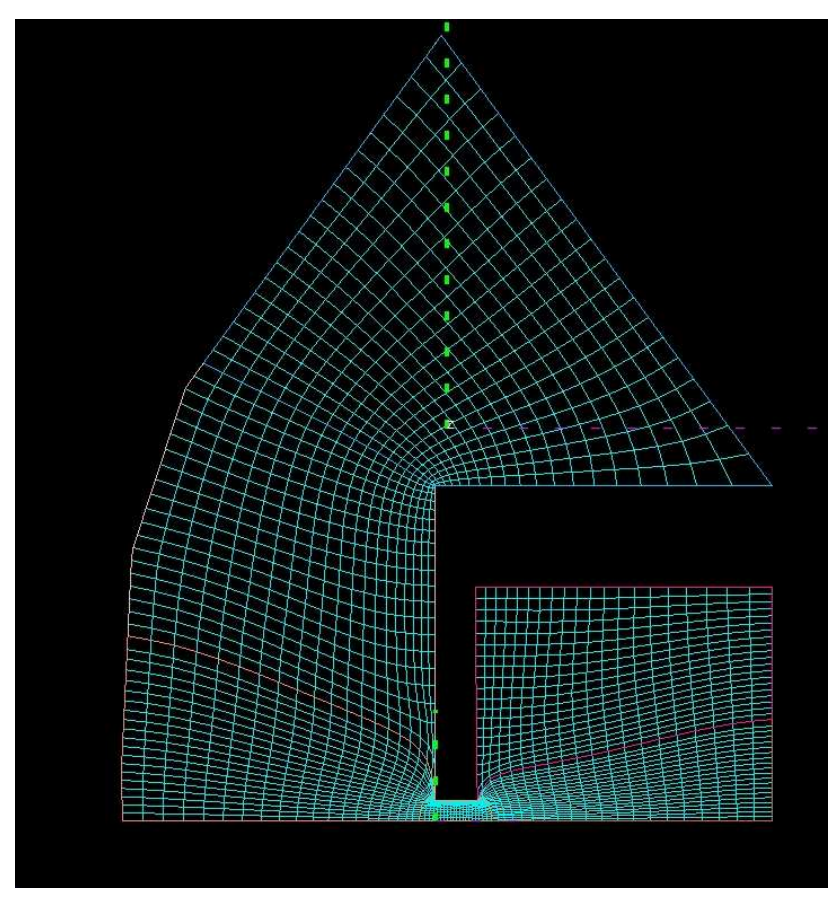

Figure 7. The simplest 1151 case mesh. Note the red color lines are the topology edges seen in Fig 6.

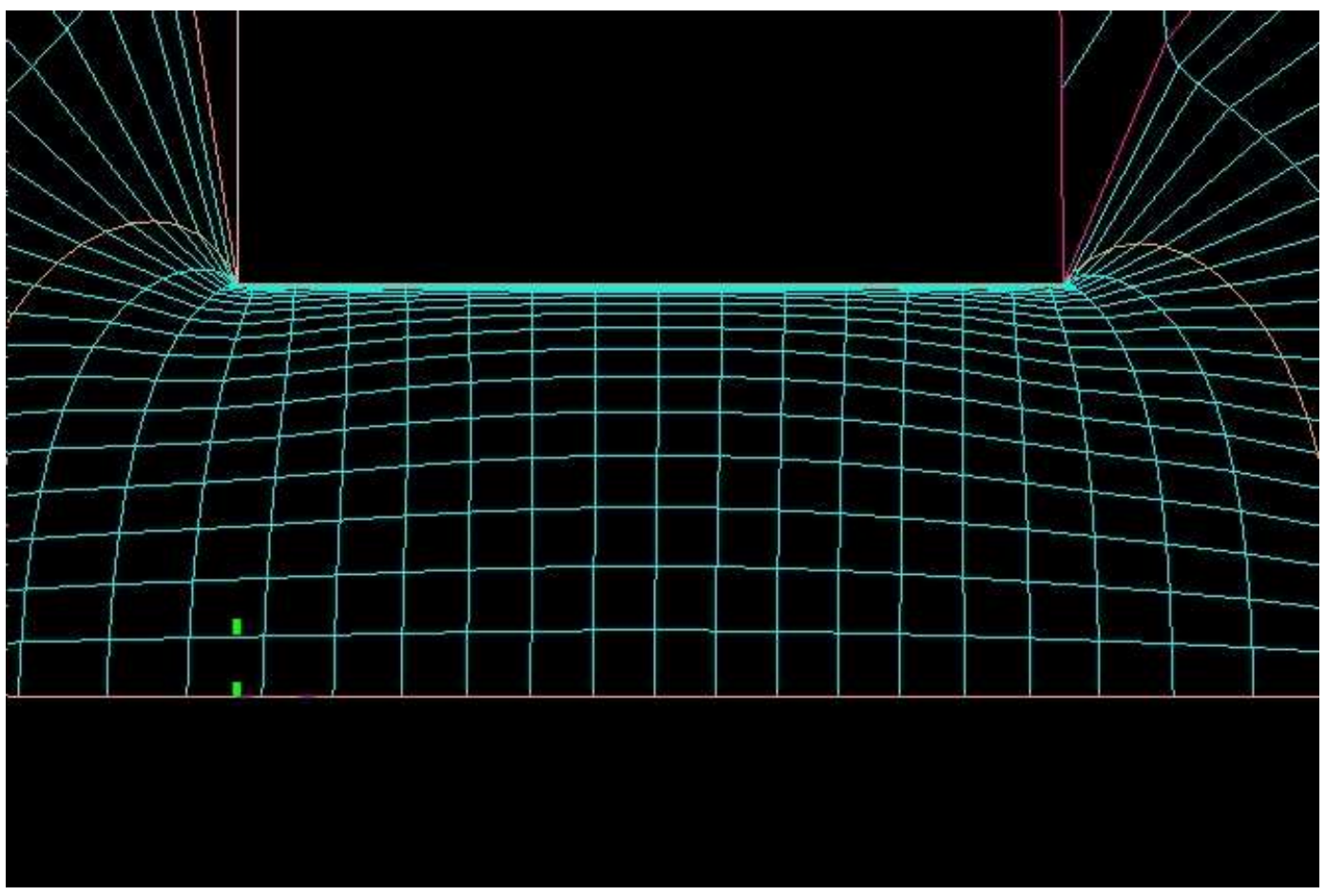

Figure 8. The simplest 1151 mesh in the channel Note the red color lines are the topology edges seen in Fig 6 


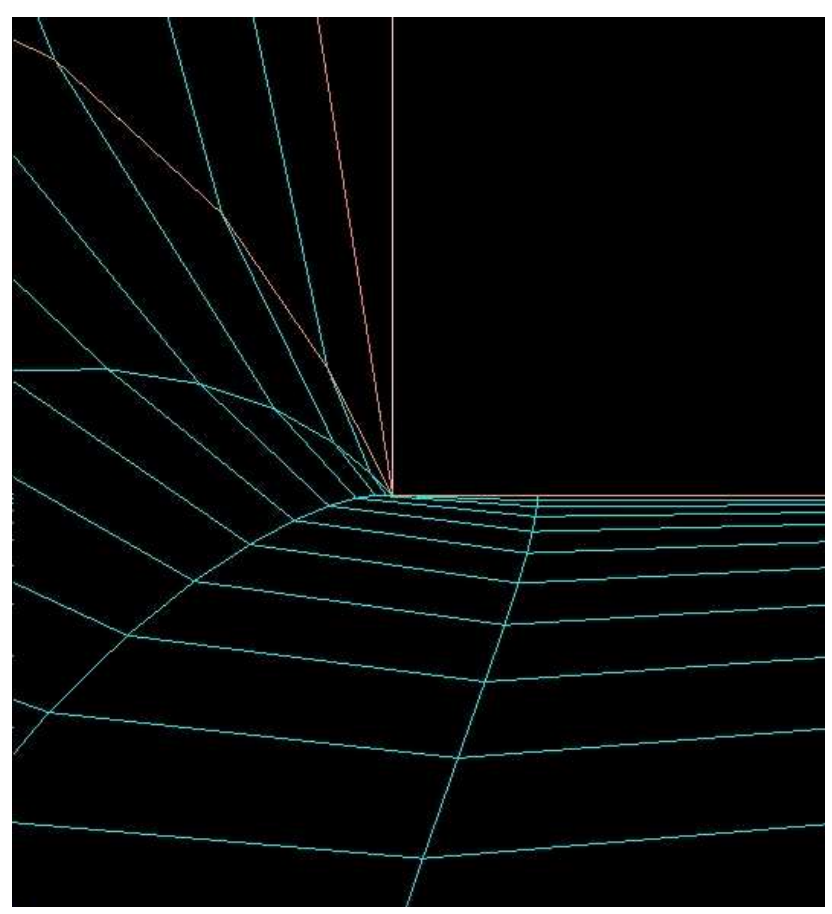

Figure 9. The simplest 1151 mesh in the corner at the entrance of the channel. The problems of the mesh can be clearly observed. The colored lines except for the grin lines representing the mesh itself represent the boundaries of the mesh blocks.

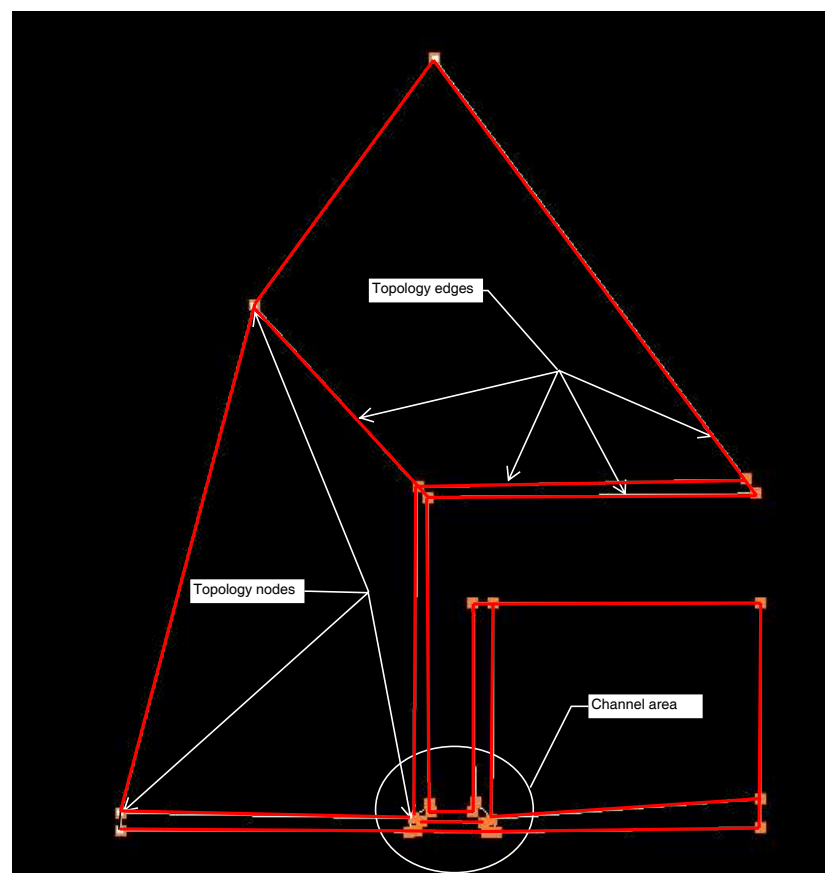

Figure 10. 1151 improved topology. This is still a 2D topology with all of the new topology nodes lying in the same plane with the original topology nodes. 


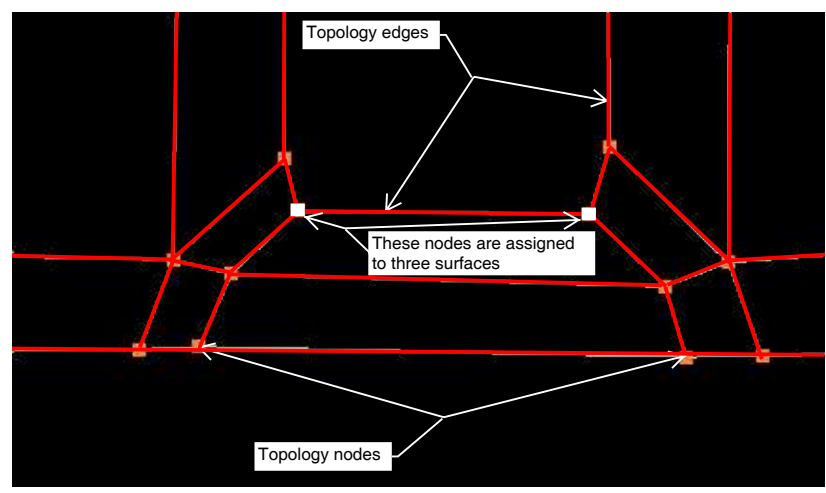

Figure 11. 1151 improved topology in the channel region.

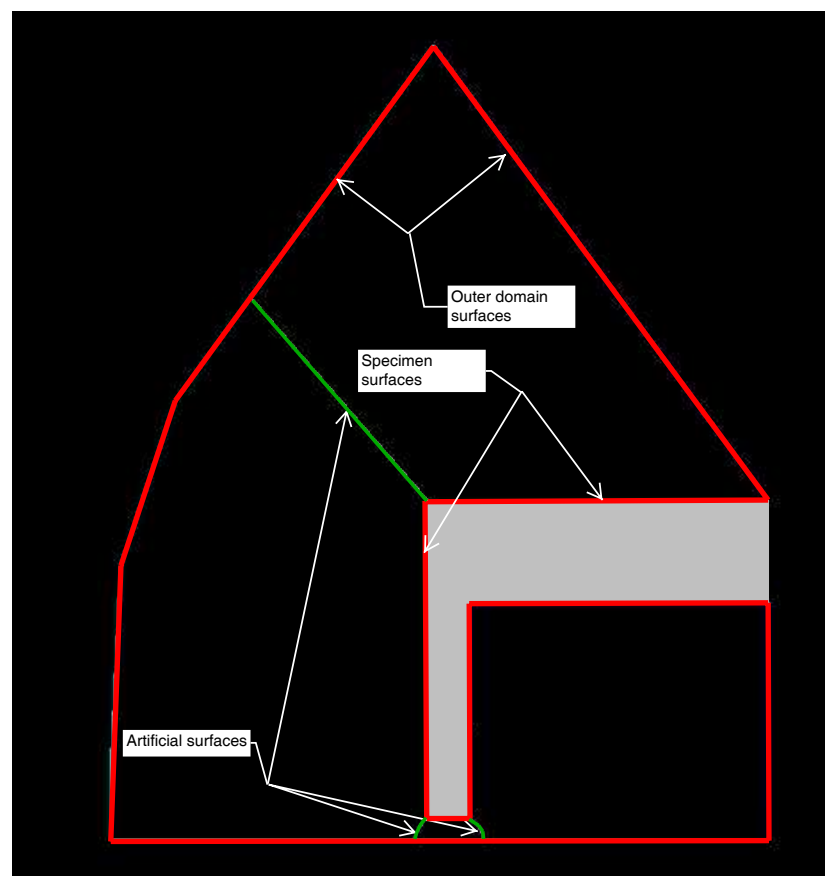

Figure 12. 1151 domain representation with artificial surfaces. 


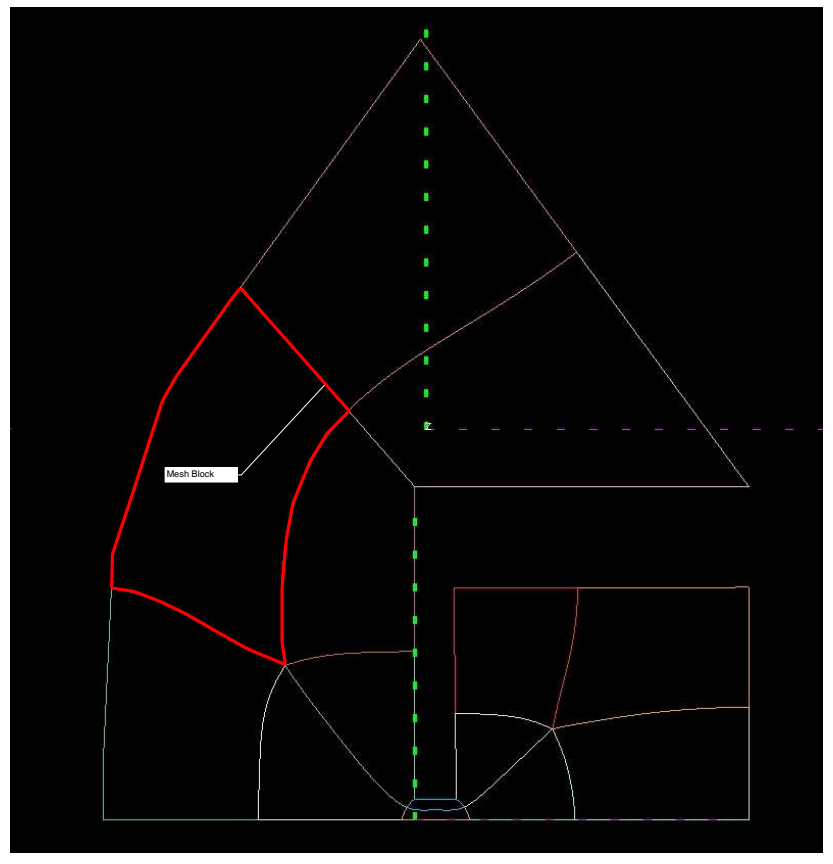

Figure 13. 1151 improved block mesh structure. The colored lines represent the boundaries of the mesh blocks. The red lines highlight the boundaries of one of the blocks.

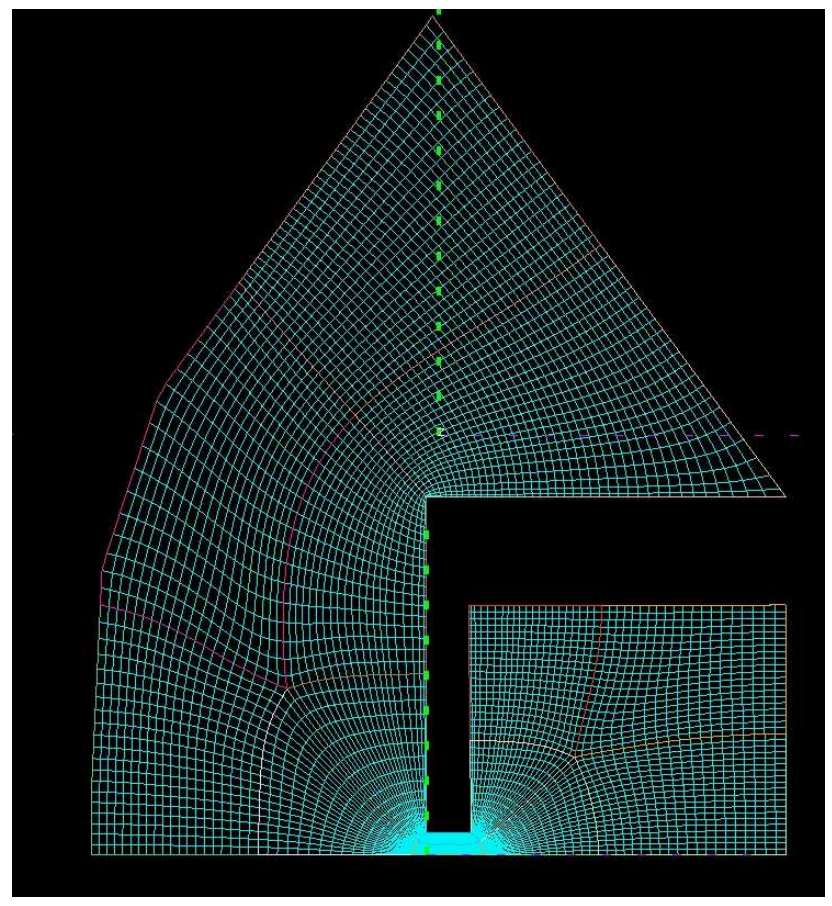

Figure 14. 1151 improved mesh. The entire domain. The colored lines, except for the green lines that represent the mesh itself, represent the boundaries of the mesh blocks. 


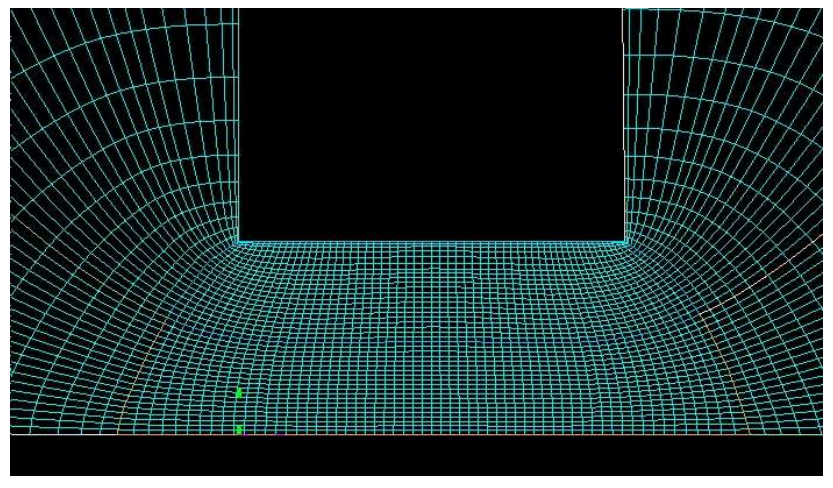

Figure 15. 1151 improved mesh. The mesh in the channel

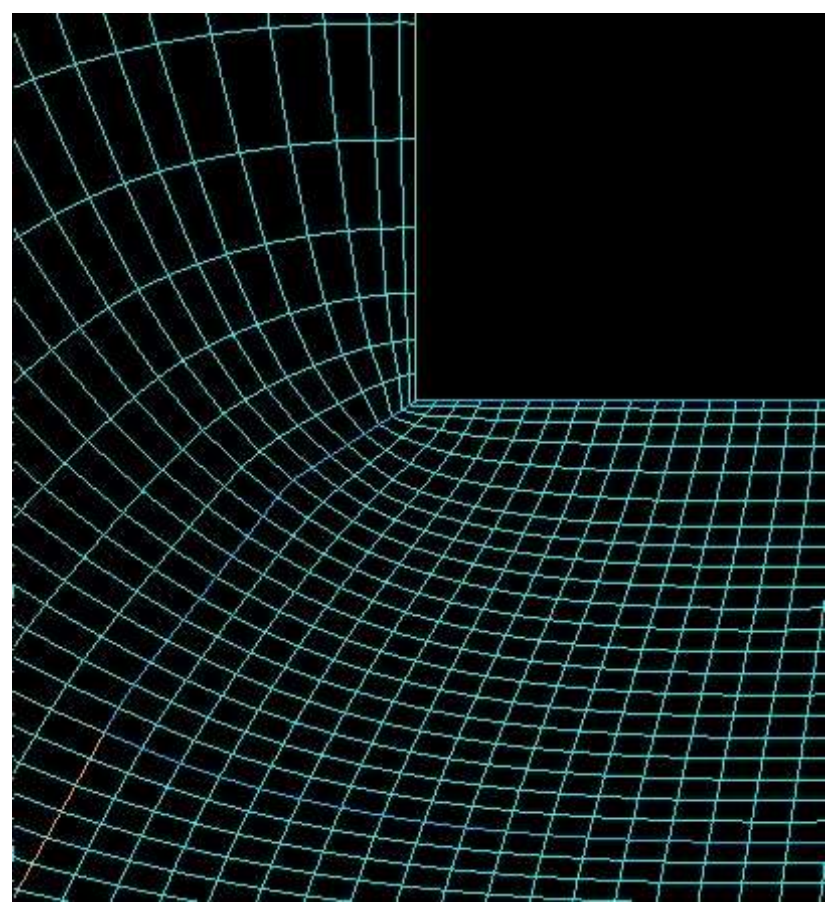

Figure 16. 1151 improved mesh. The mesh at the corner of the channel entrance. Most of the mesh problems are resolved. 


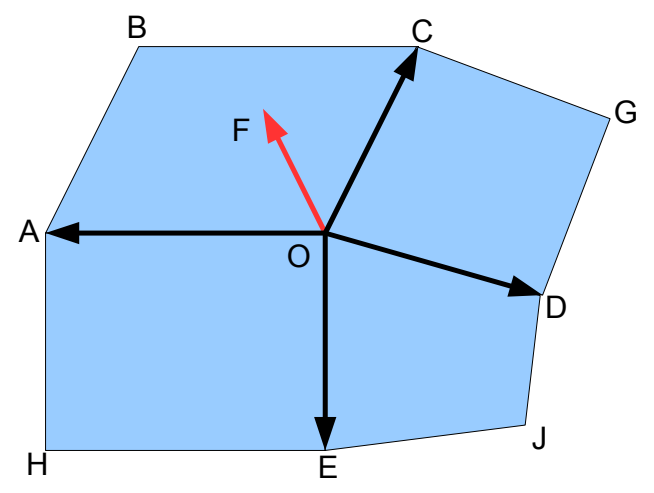

Figure 17. The surface normal definition. Point $O$ represents a surface node shared by four surface quadrilaterals, ABCO, CGDO, ODJE and OEHA. Note that point $F$ is not in the plane of any of quads.

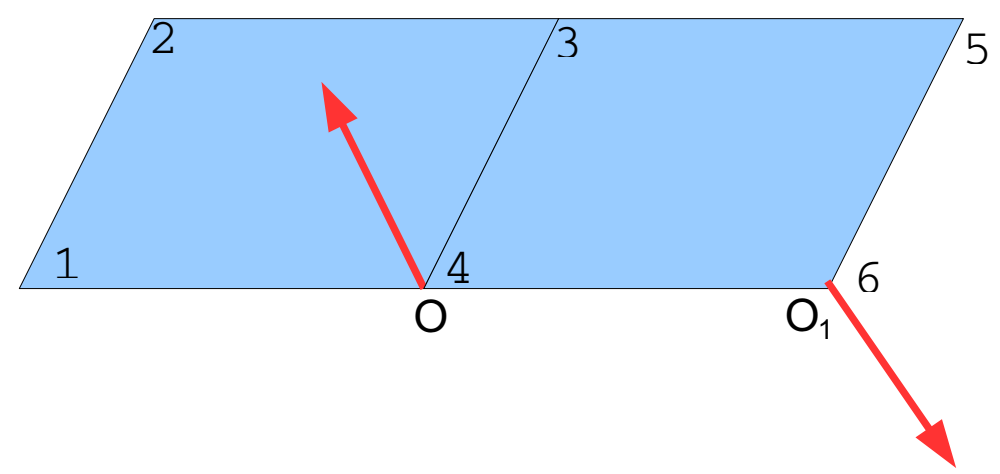

Figure 18. The normal directions with respect to the surface side (with indicated in red) are undefined. 


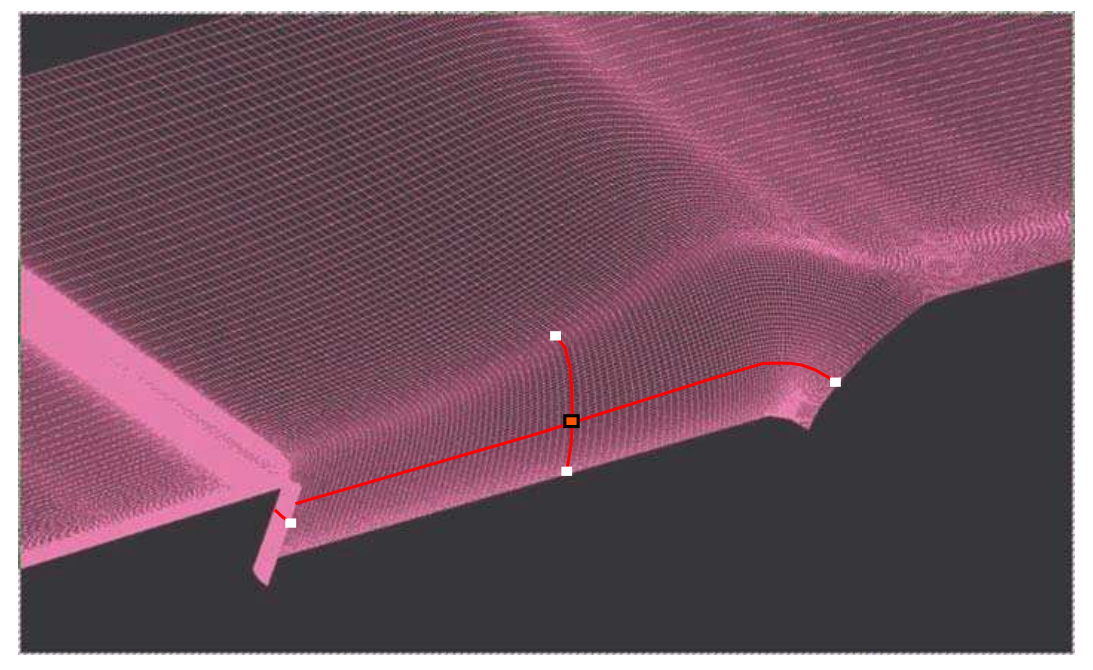

Figure 19. The analytical approximation of the channel surface

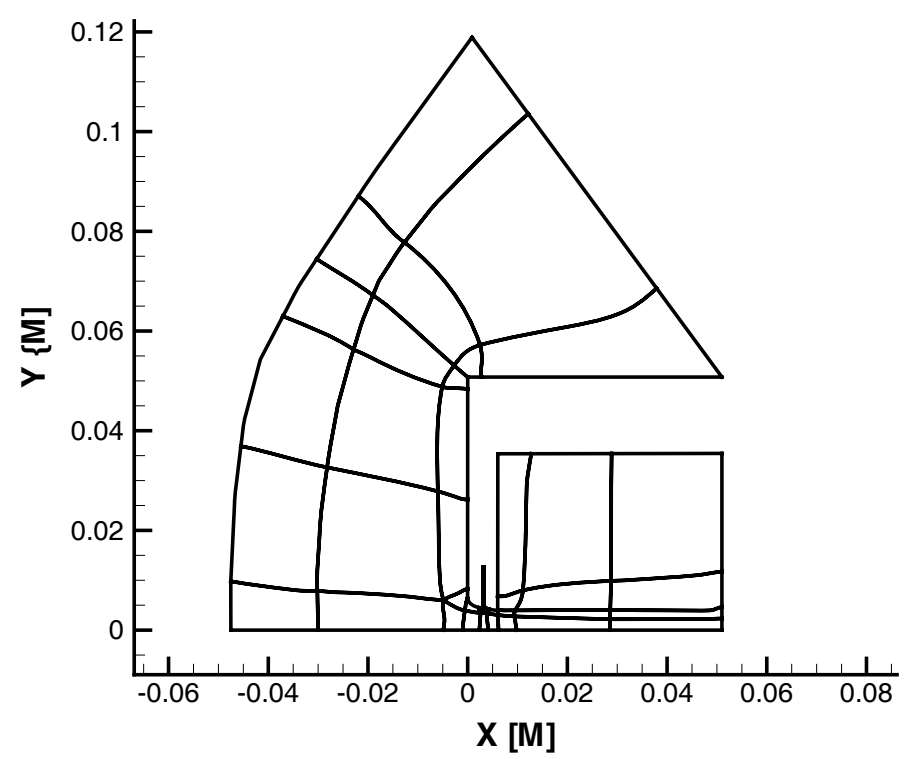

Figure 20. Multi zone layout of the computational domain. The presented case corresponds to 50 sec. 


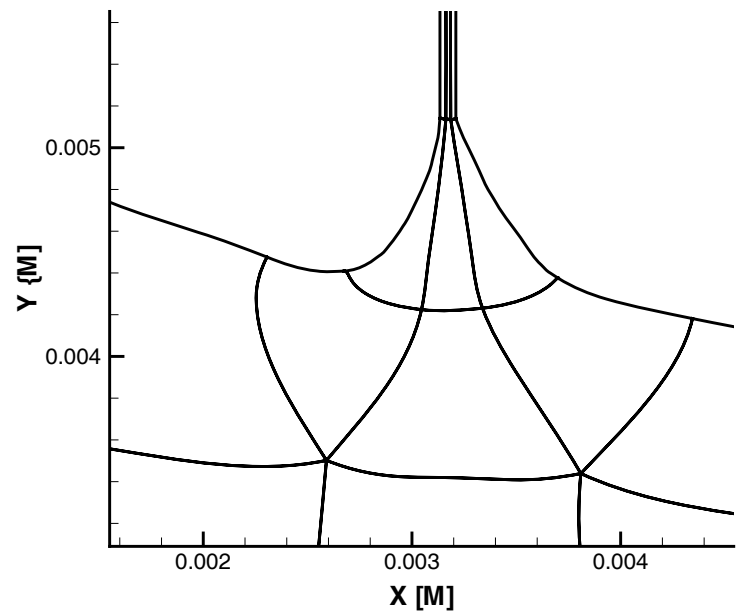

Figure 21. The domain layout in vicinity of the sub channel. 


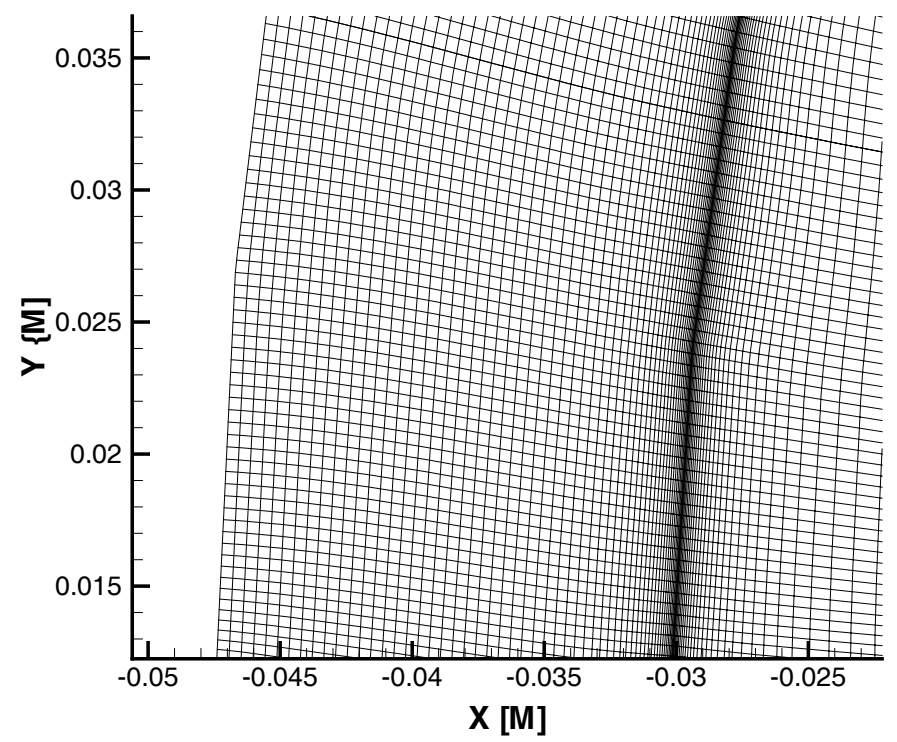

Figure 22. Mesh adaptation in the shock wave area allowed for a better gradient resolution.

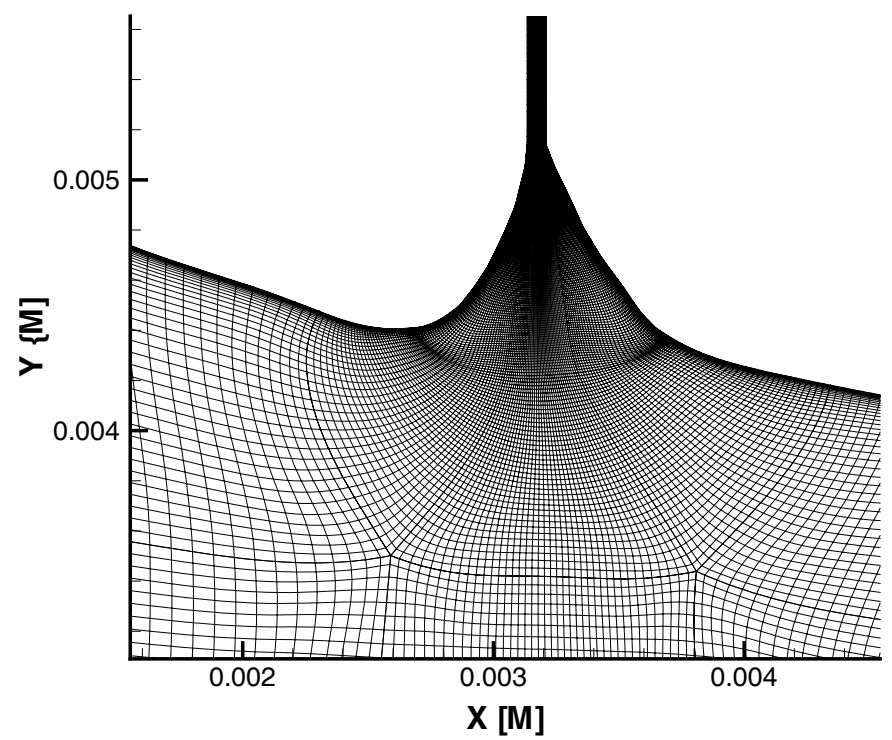

Figure 23. The sub-channel entrance meshing. Mesh clustering close to the wall is presented along with the overall quality of the mesh in vicinity of the sub-channel entrance. The presented case corresponds to 50 sec. 


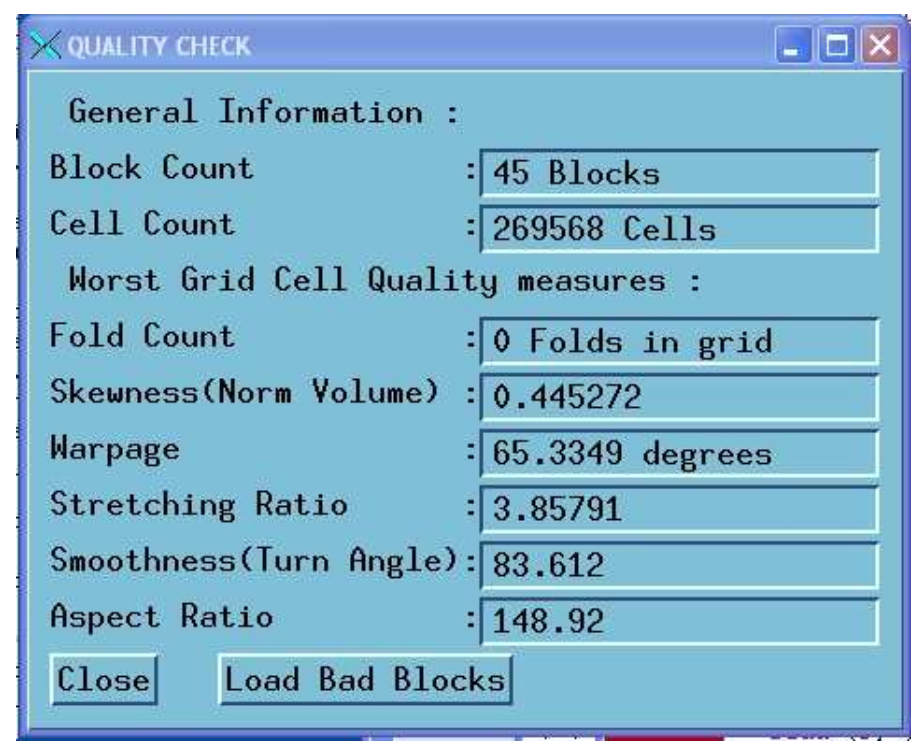

Figure 24. Mesh goodness testing information produced by Gridpro.

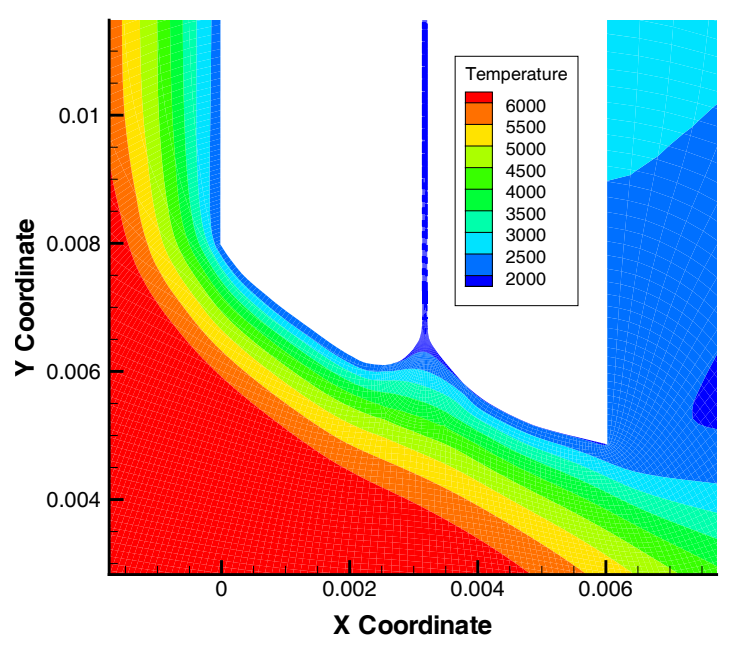

Figure 25. Temperature contours in the channel. [K] 


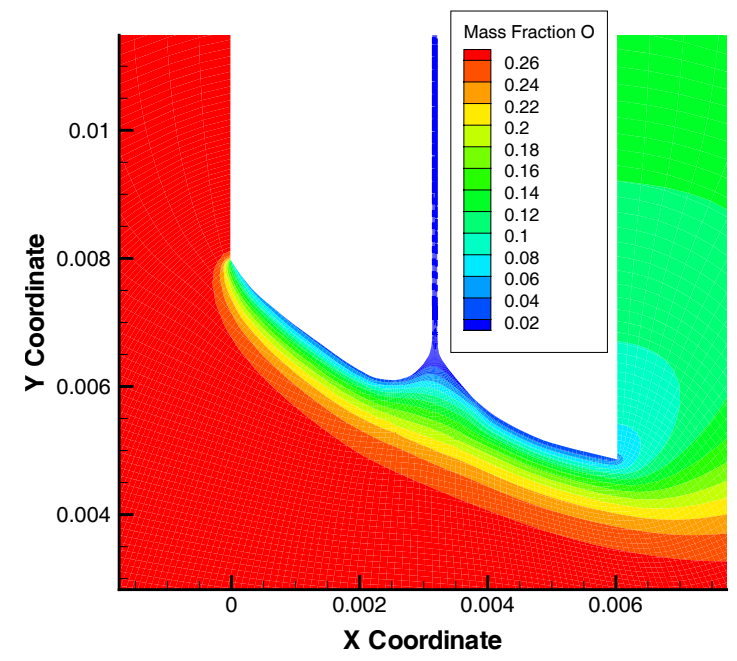

Figure 26. Oxygen mass fraction in the channel.

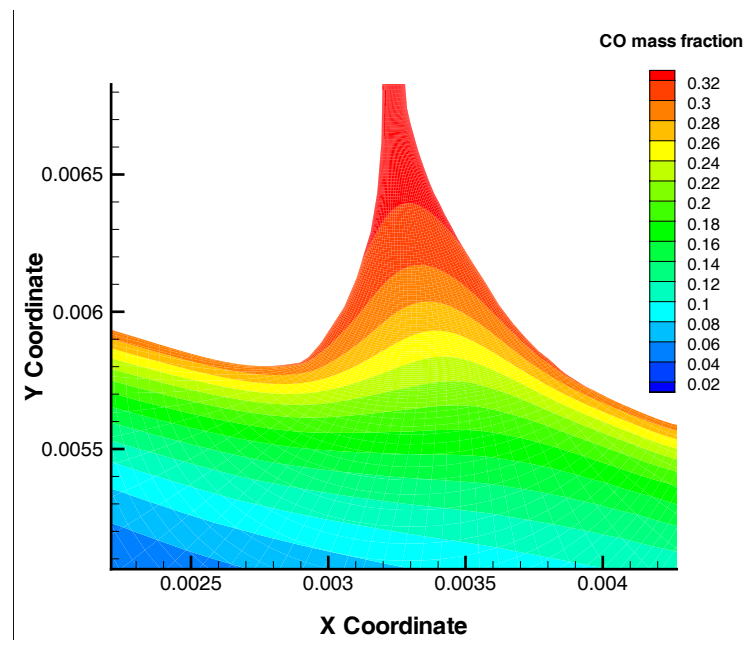

Figure 27. CO mass fraction contours in the channel. 


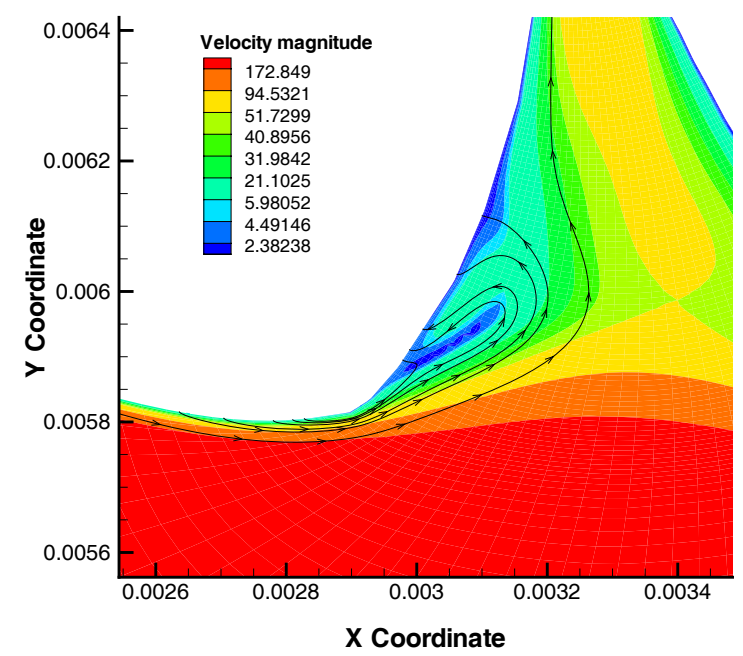

Figure 28. Velocity streamlines. Colors present velocity magnitudes in the channel. [m/s]

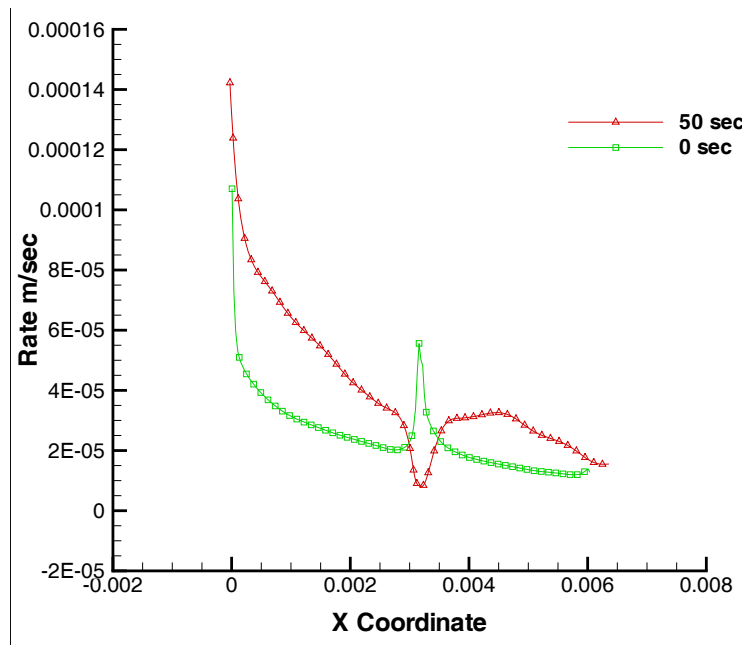

Figure 29. Oxidation rates at 0 and 50 seconds. $[\mathrm{m} / \mathrm{s}]$ 


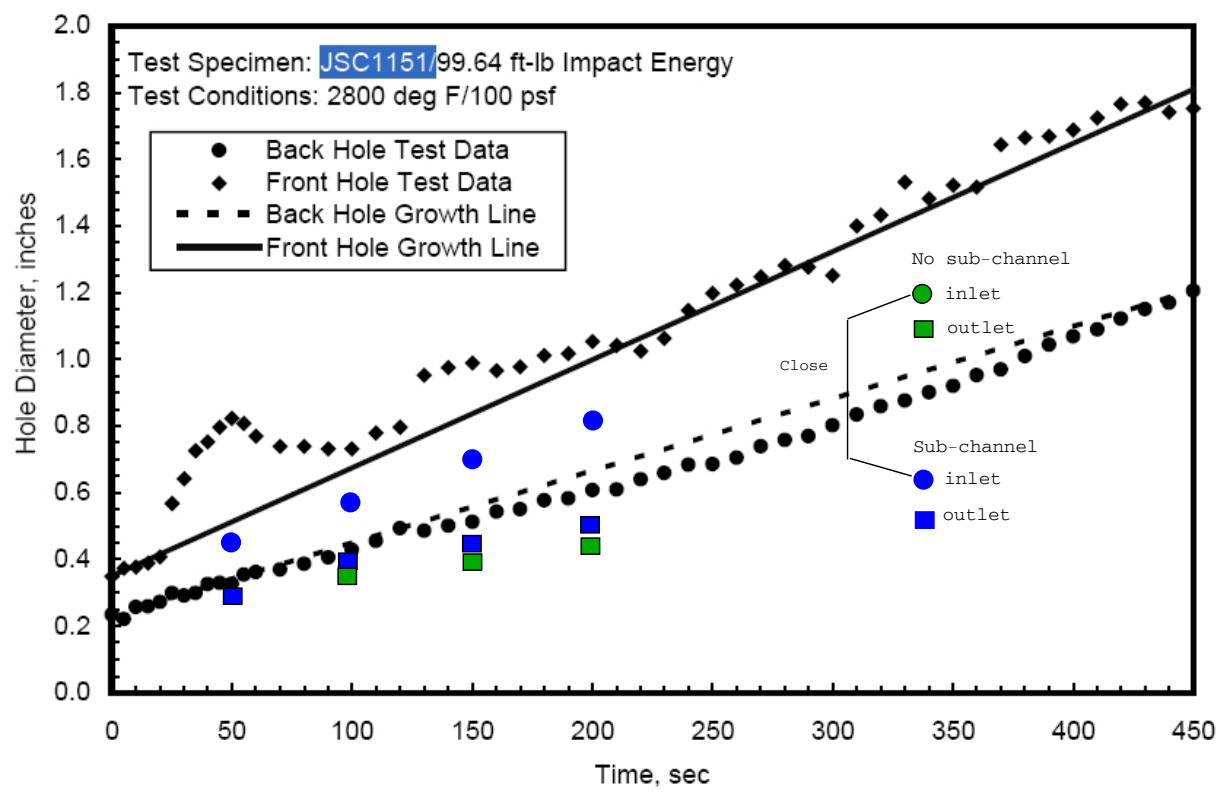

Figure B-9 Test Data of An Impacted RCC Hole Growth

Figure 30. Wall regression. Entrance and exit hole diameters. N-S results with and without sub-channel. $[\mathrm{m} / \mathrm{s}]$

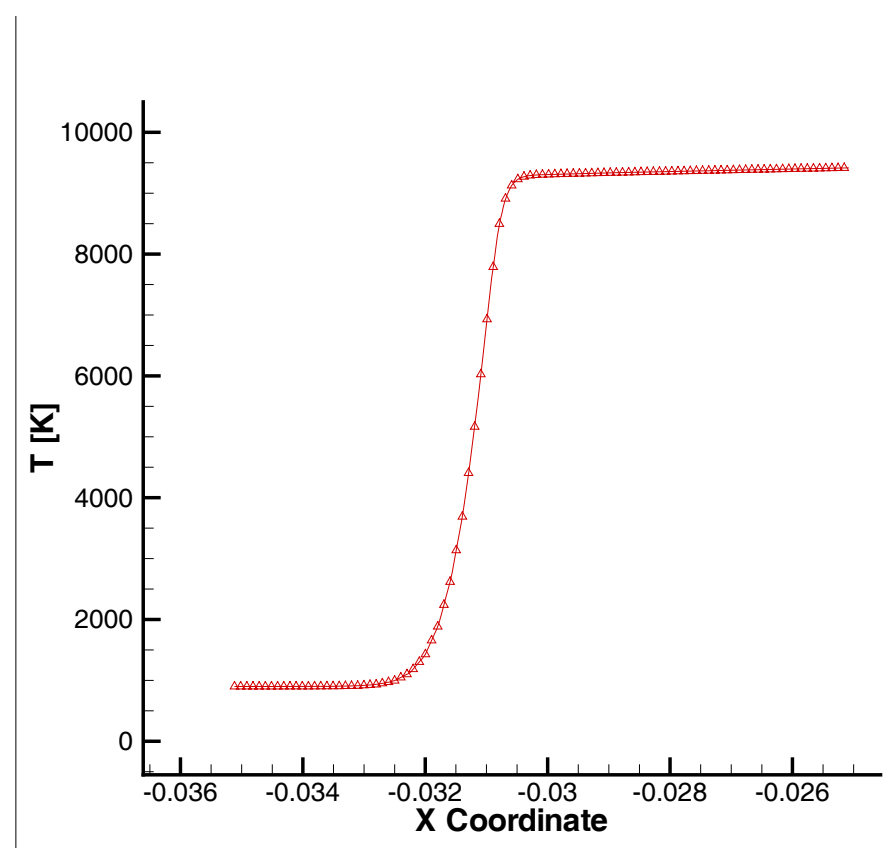

Figure 31. Temperature in the shock. [K] 


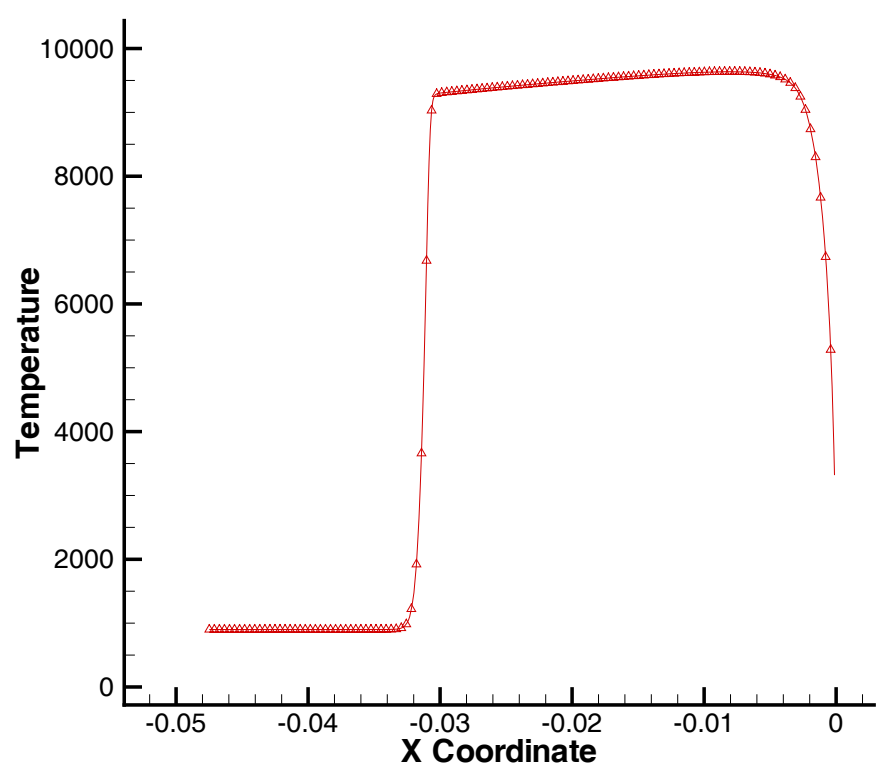

Figure 32. Temperature along the stagnation line. [K]

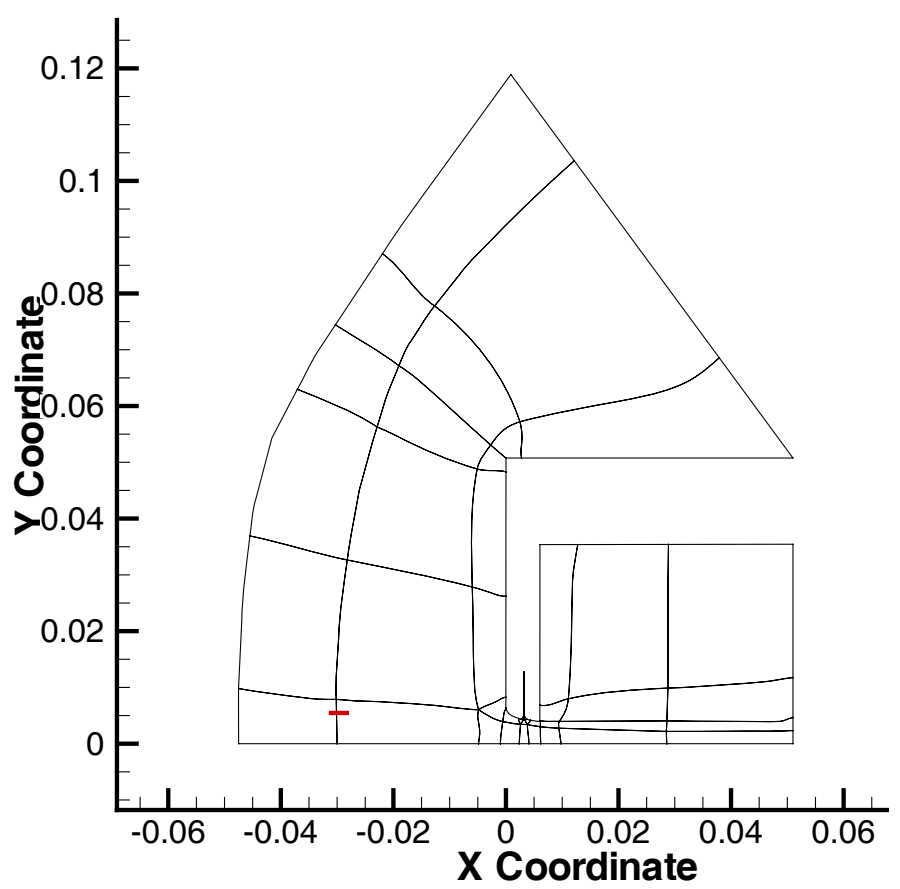

Figure 33. Location of the shock sample line 


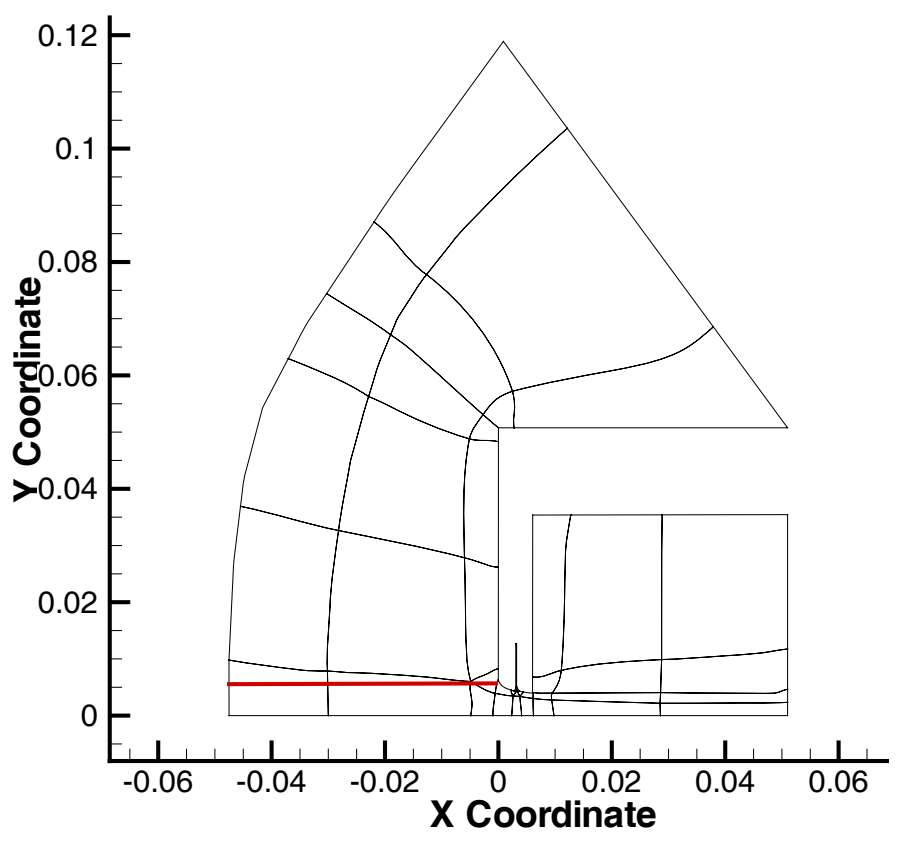

Figure 34. Location of the stagnation sample line

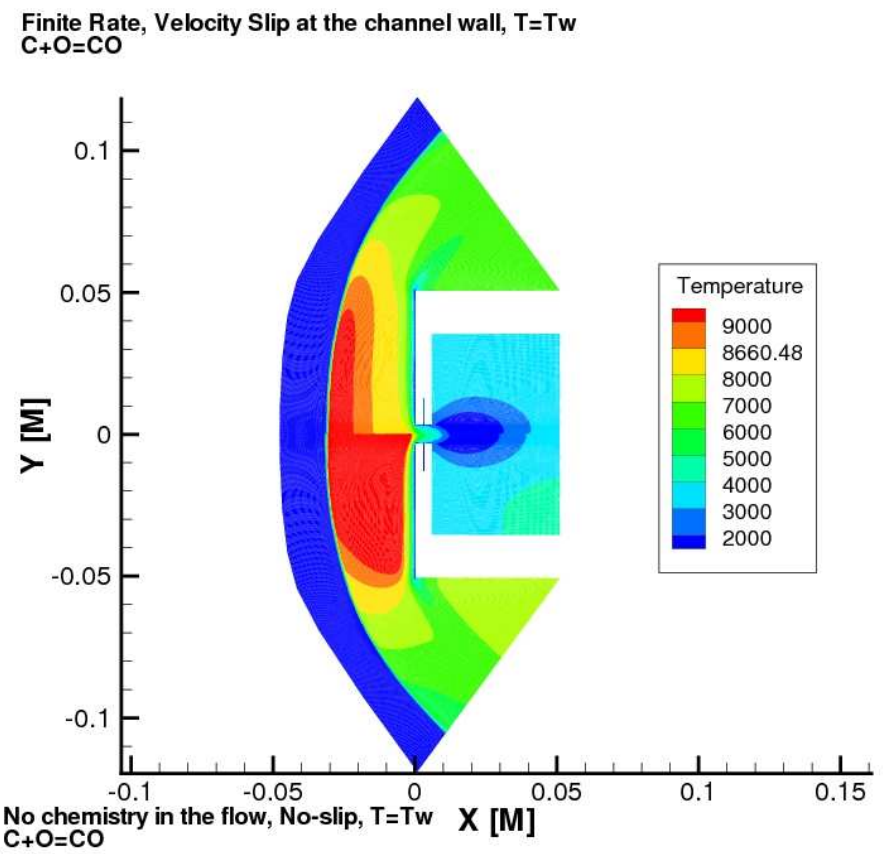

Figure 35. Temperature flow fields with and without flow chemistry $[\mathrm{K}]$. 


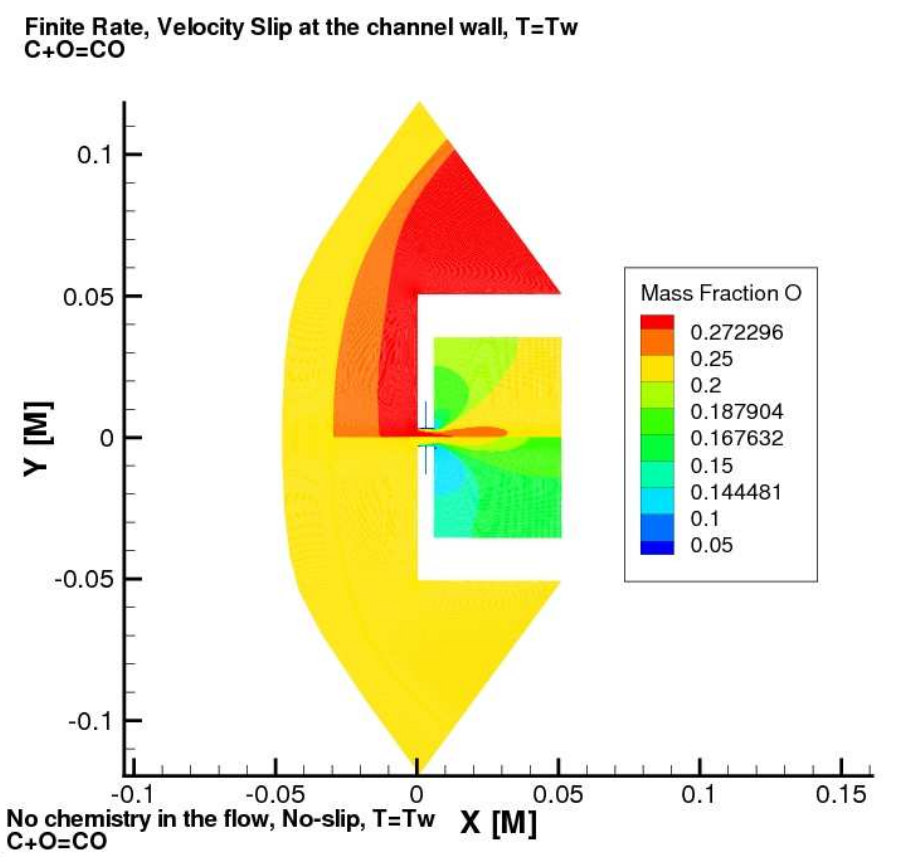

Figure 36. Oxygen mass fraction flow fields with and without flow chemistry.

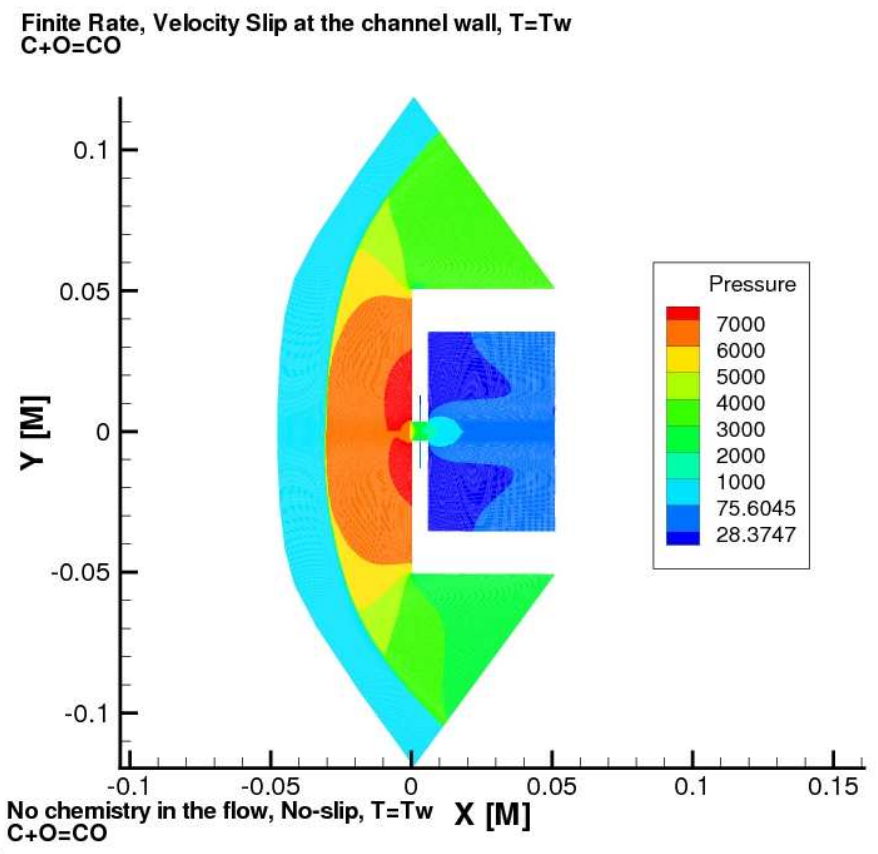

Figure 37. Pressure flow fields with and without flow chemistry $[\mathrm{Pa}]$. 


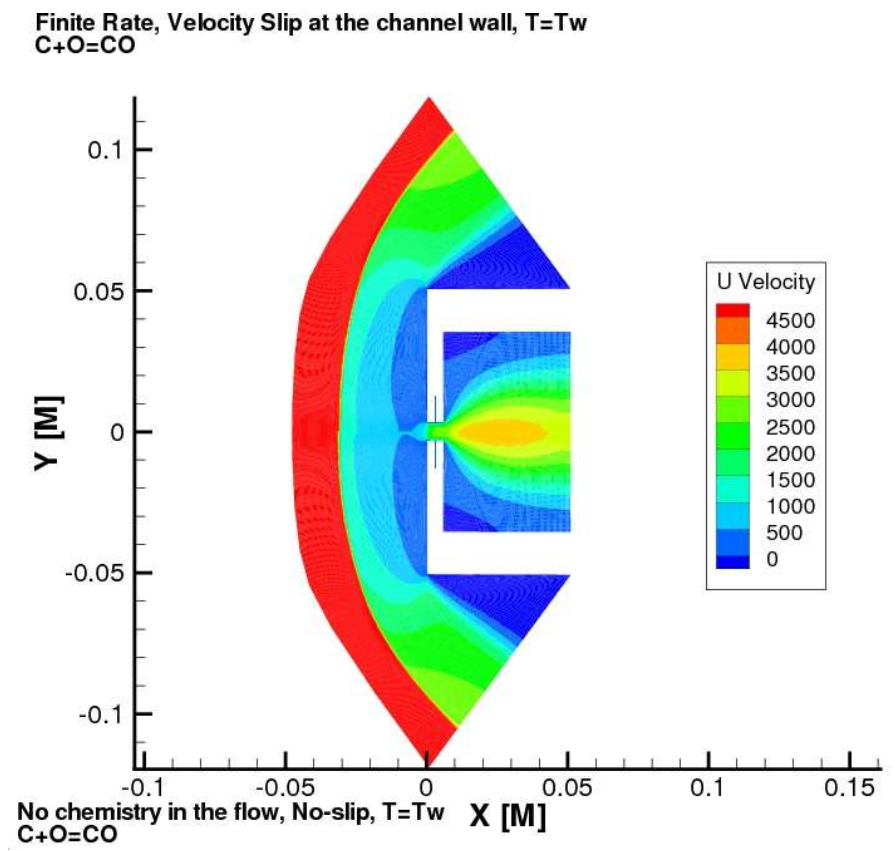

Figure 38. X component velocity fields with and without flow chemistry $[\mathrm{m} / \mathrm{s}]$.

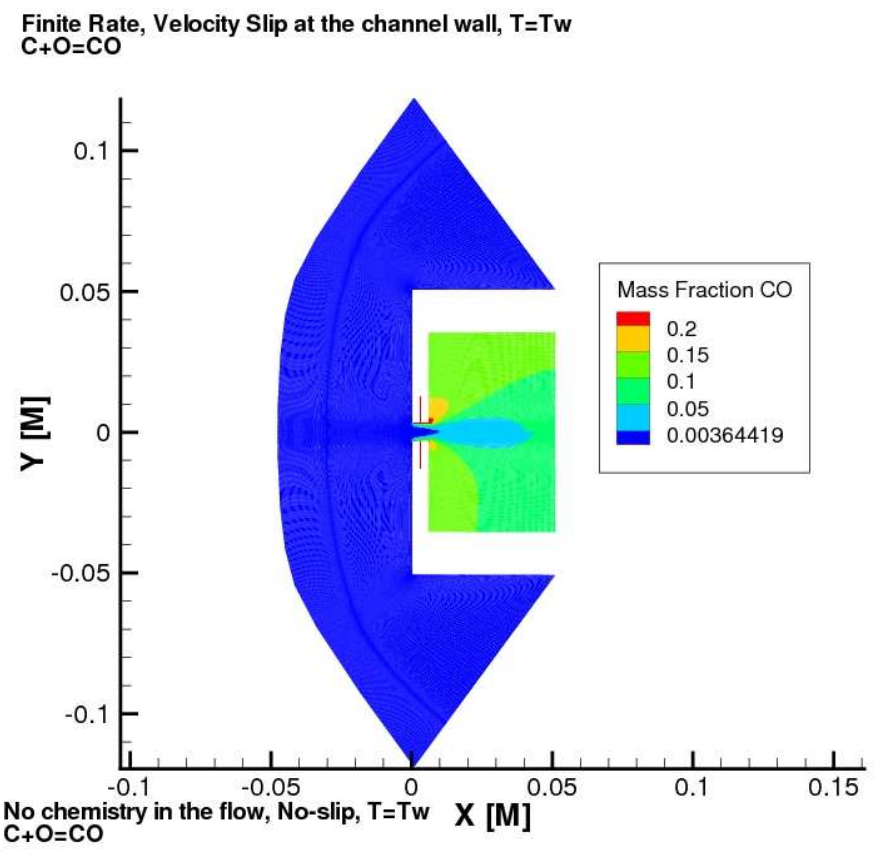

Figure 39. CO mass fraction flow fields with and without flow chemistry. 


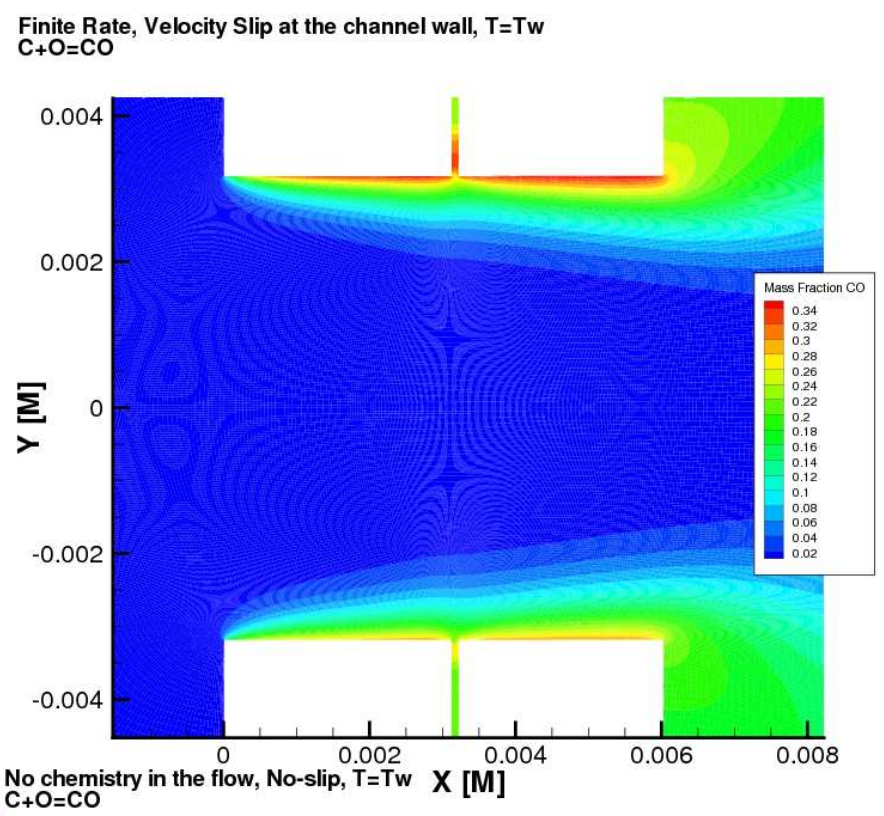

Figure 40. CO mass fraction flow fields in the channel with and without flow chemistry.

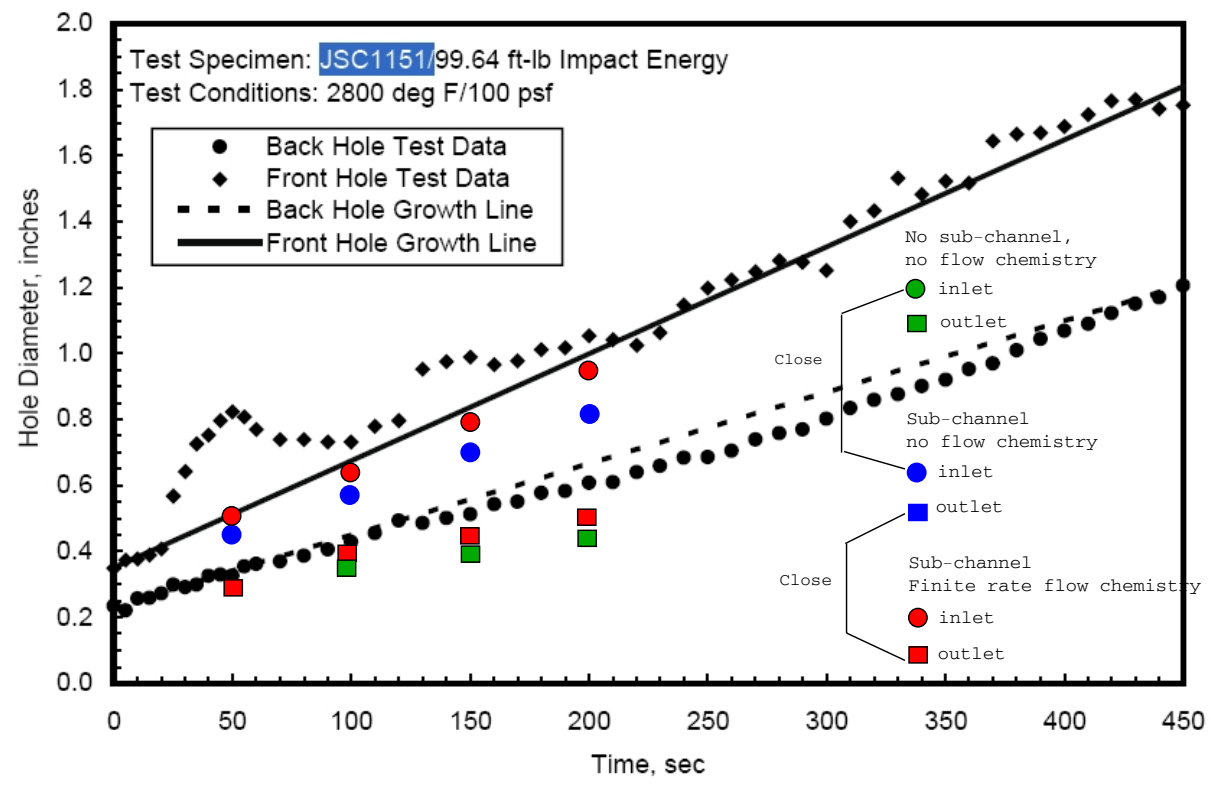

Figure B-9 Test Data of An Impacted RCC Hole Growth

Figure 41. Wall regression. Entrance and exit hole diameters. N-S with and without chemistry in addition to with and without sub-channel. $[\mathrm{m} / \mathrm{s}]$ 


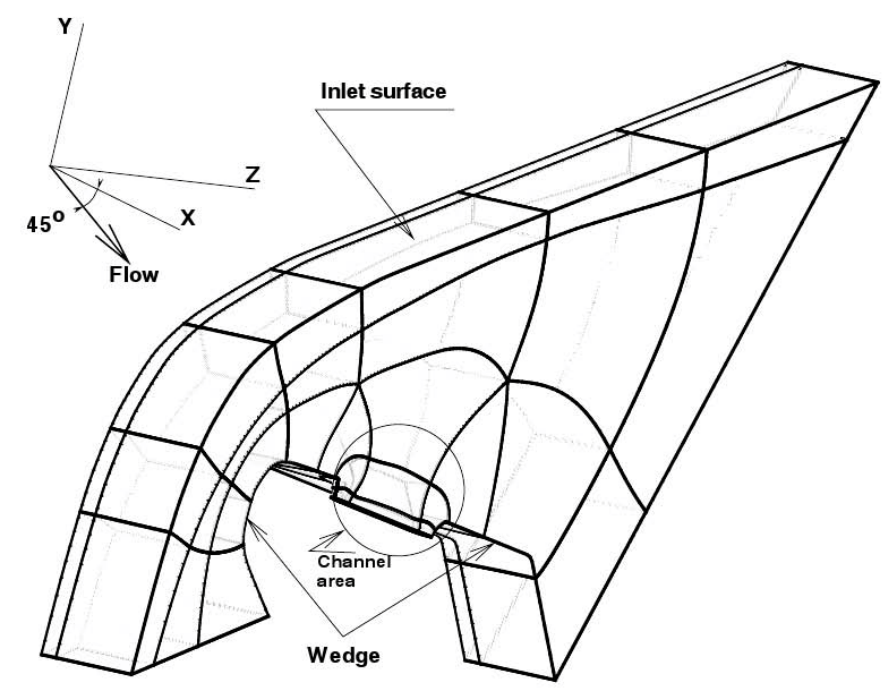

Figure 42. 320 sec case. Topology of the computational domain.

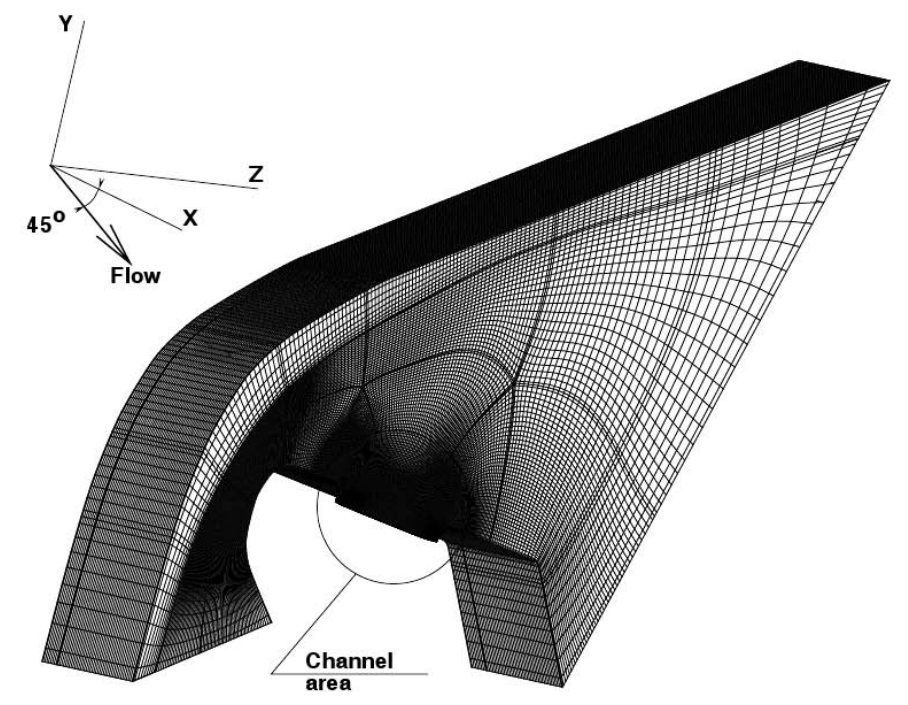

Figure 43. 320 sec case. Computational mesh. 


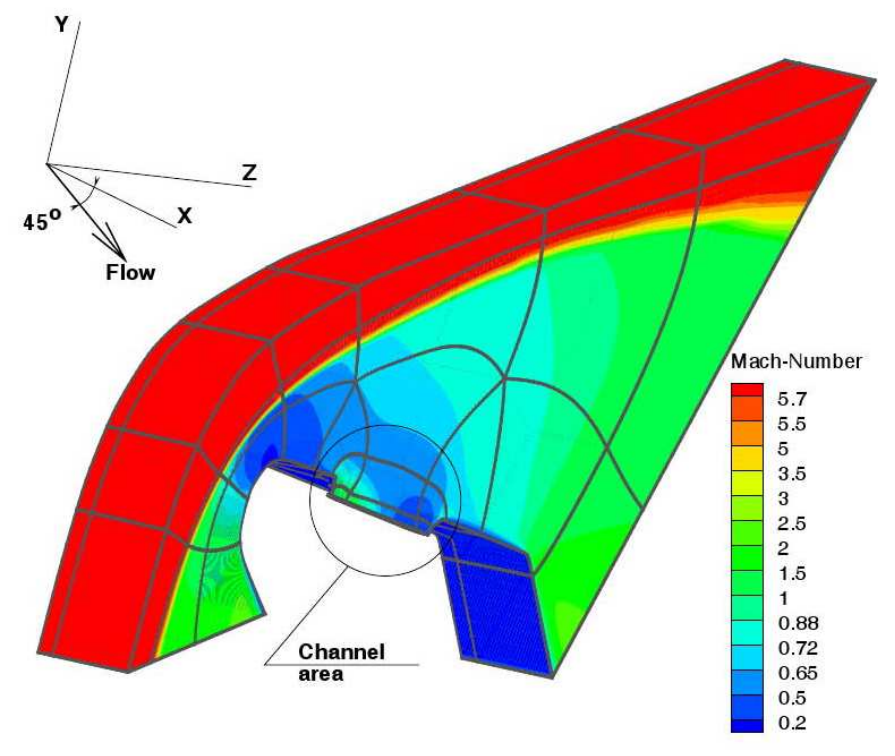

Figure 44. 320 sec case Mach number contours in the 3D computational domain.

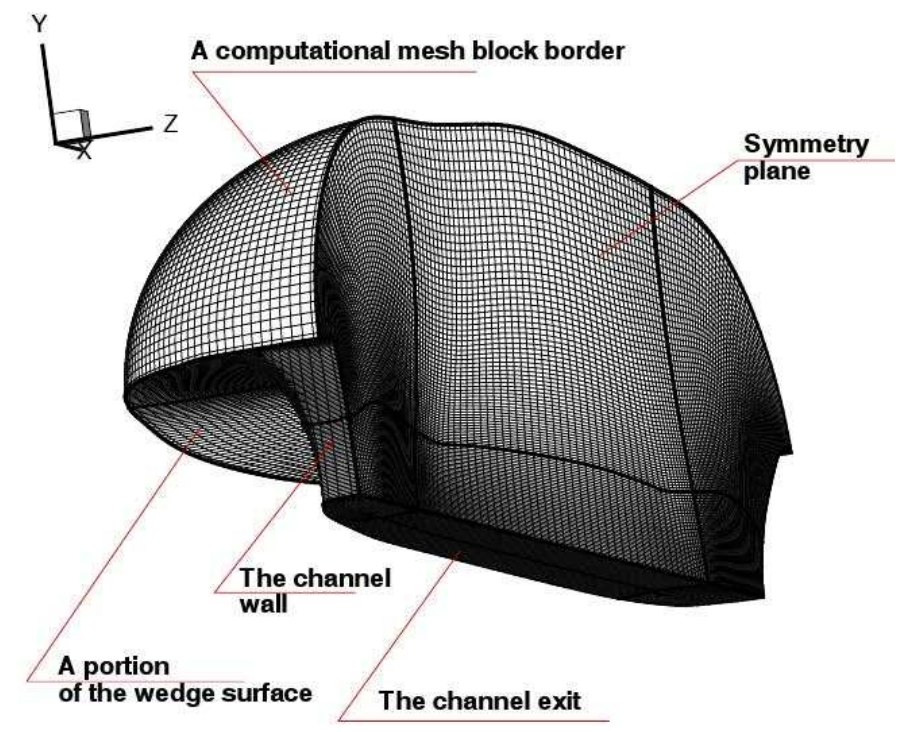

Figure 45. 320 sec case. An excerpt of the computational mesh in vicinity of the channel area. 


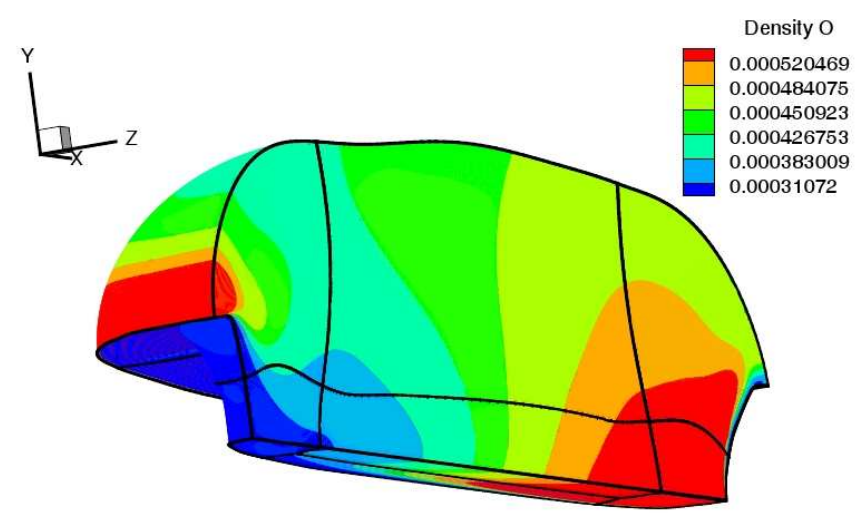

Figure 46. 320 sec case. Atomic oxygen number density in vicinity of the channel.

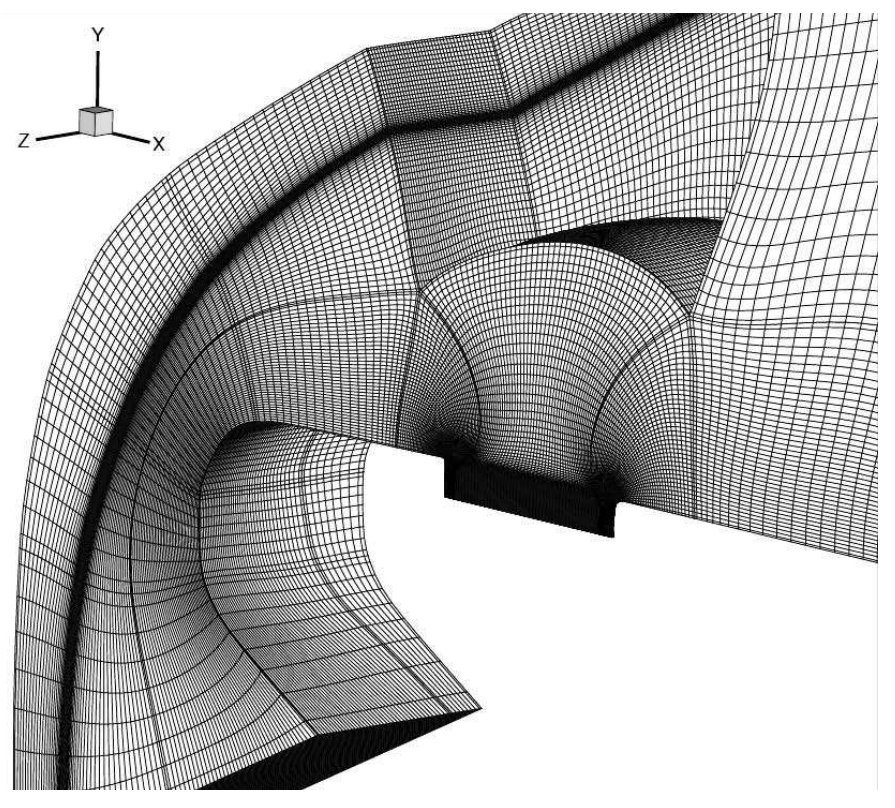

Figure 47. 60 sec case. Computational mesh. Illustration of the shock wave capturing. 


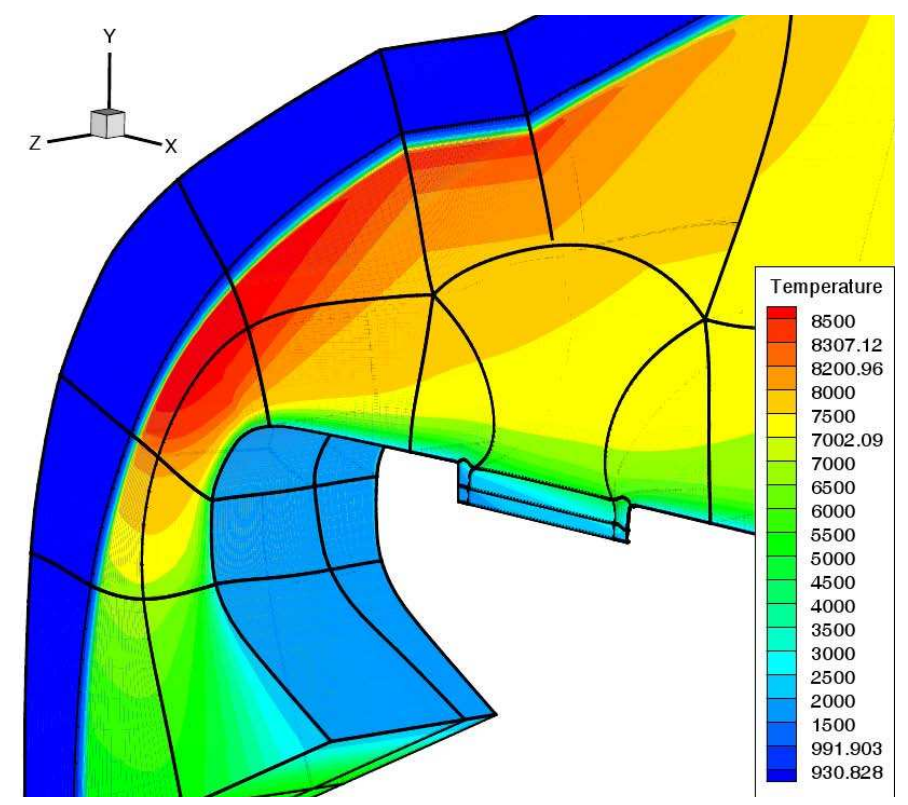

Figure 48. 60 sec case. Temperature contours in the 3D computational domain. 3D shock wave capturing.

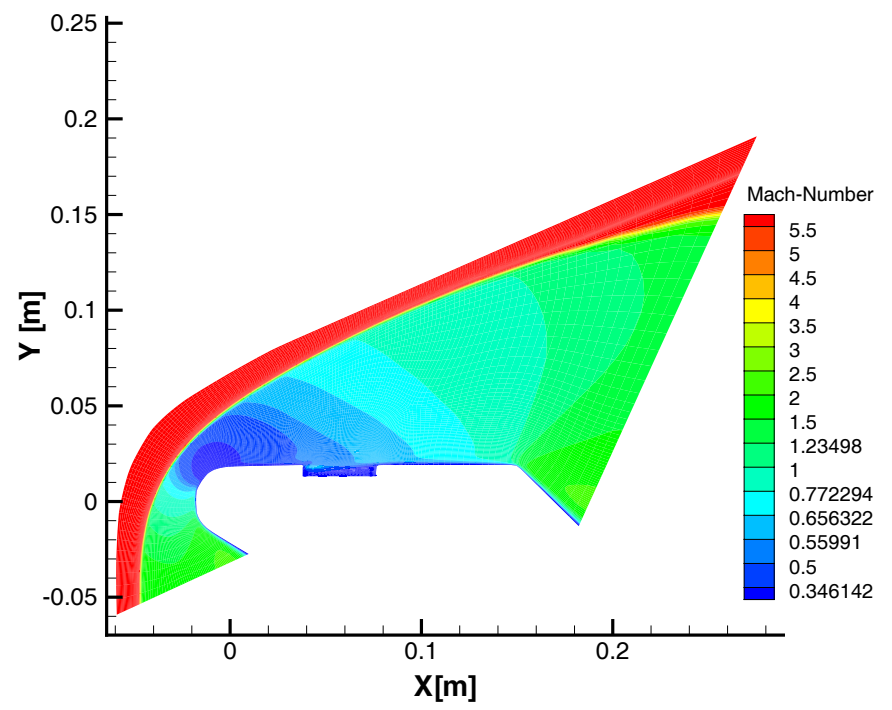

Figure 49. 60 sec case. Mach number contours in the symmetry plane. 


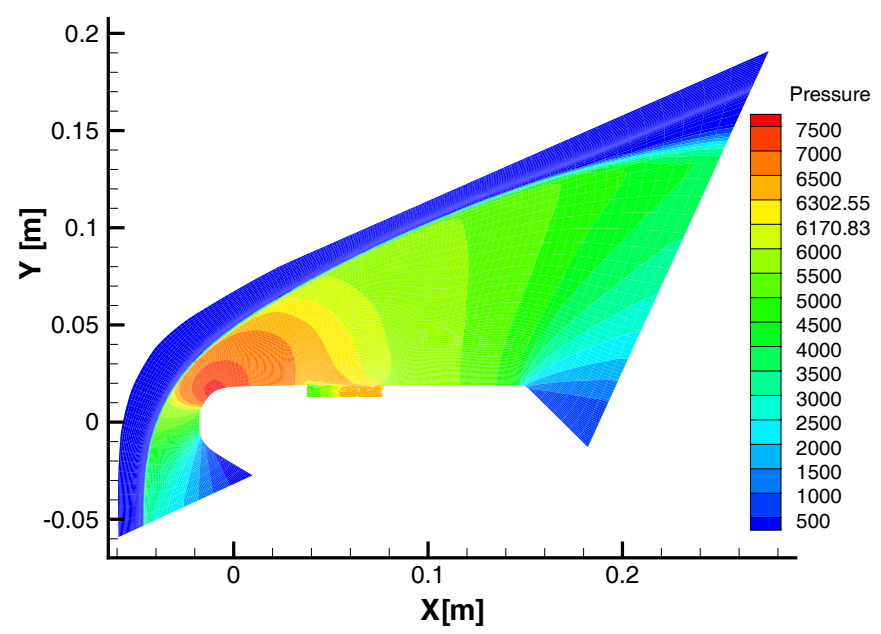

Figure 50. 60 sec case. Pressure contours in the symmetry plane.

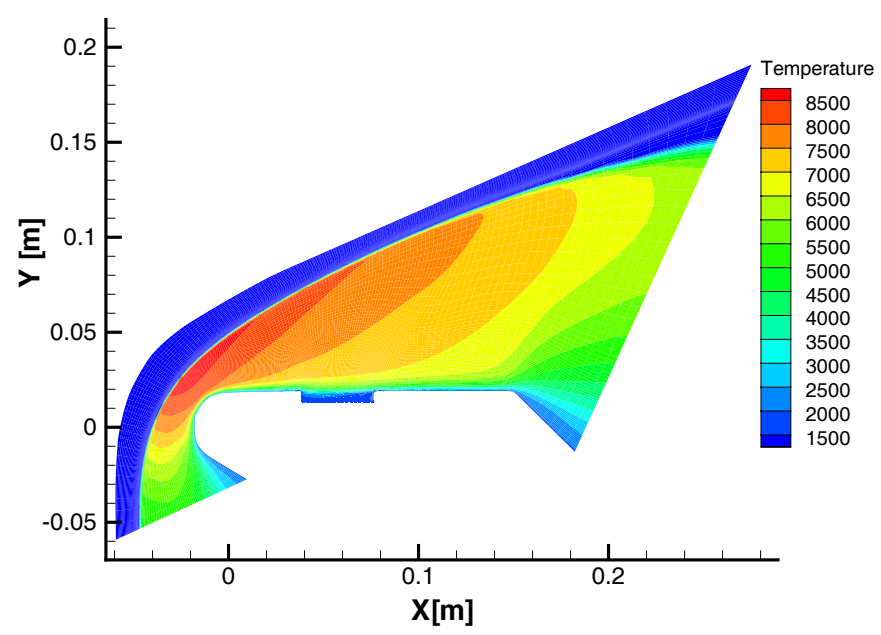

Figure 51. 60 sec case. Temperature contours in the symmetry plane.

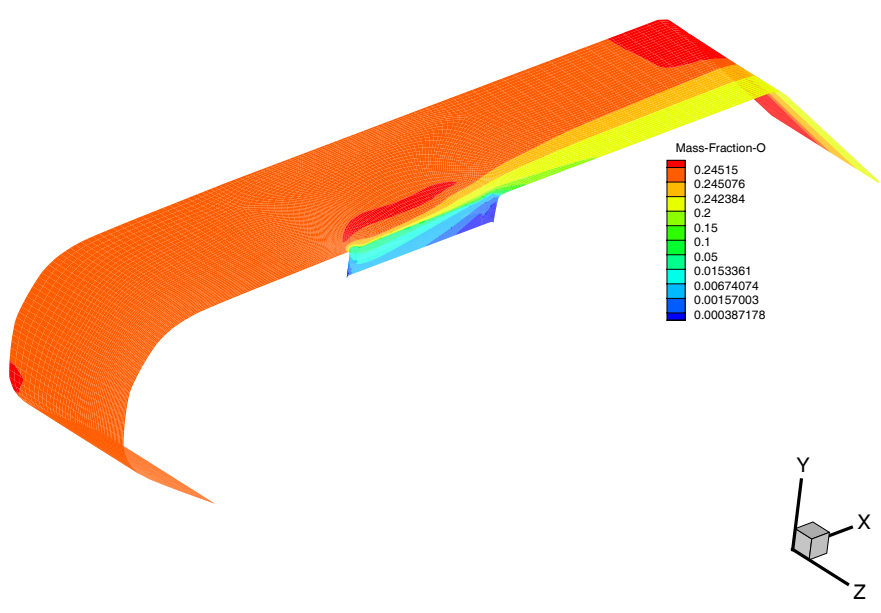

Figure 52. 60 sec case. O Mass fraction over the specimen. 


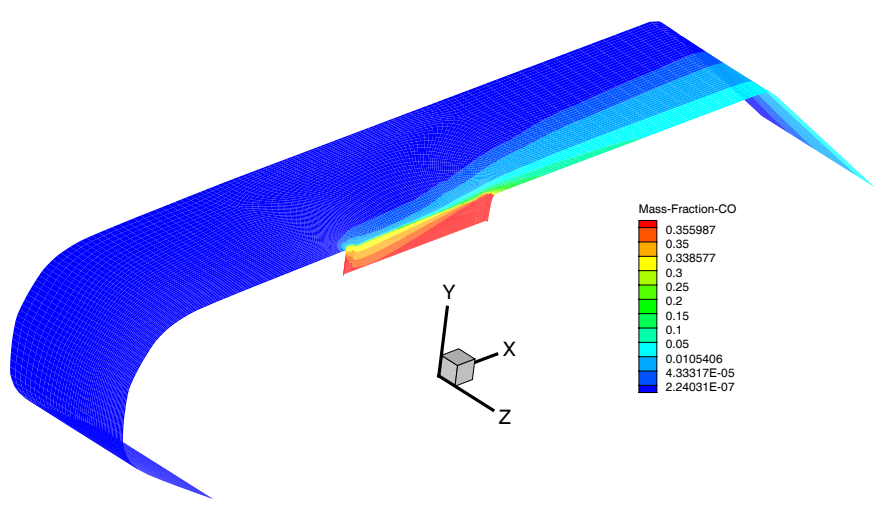

Figure 53. 60 sec case. CO Mass fraction over the specimen.

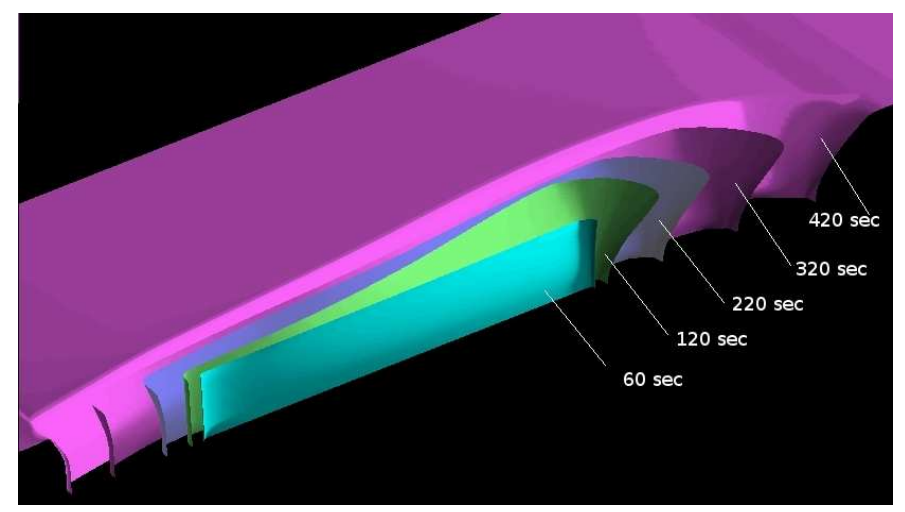

Figure 54. Channel shape at different times.

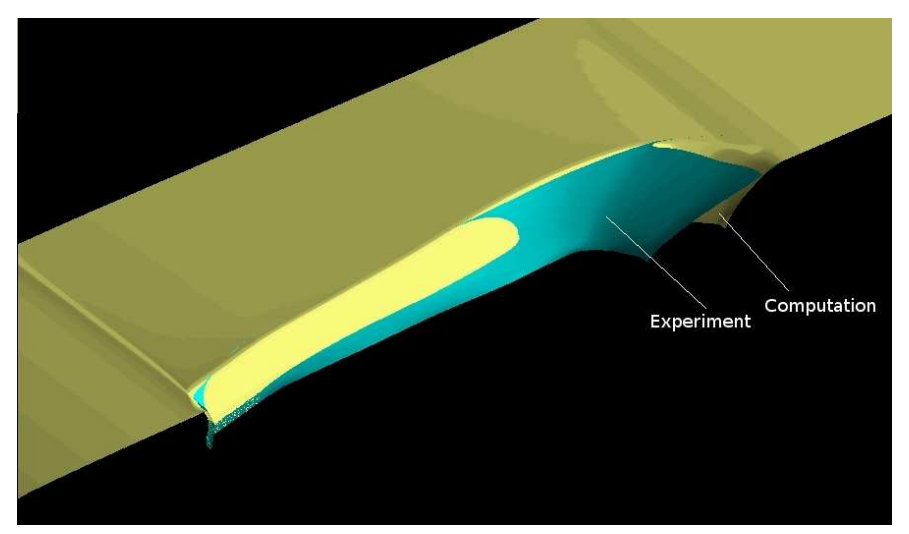

Figure 55. Comparison of the computationally obtained and the the post-test shapes of the channel. 